\title{
Competencias Digitales en Docentes de Educación Superior: Niveles de Dominio y Necesidades Formativas
}

\author{
Digital Competences in Higher Education Faculty: Levels of Mastery and \\ Training Needs \\ Competências digitais em docentes de Educação Superior: Níveis de Domínio e \\ Necessidades Formativas
}

Katia V. Pozos Pérez https://orcid.org/0000-0003-2780-4538

Grupo CIFO (Colectivo de Investigación en Formación Ocupacional)

Departamento de Pedagogía Aplicada, Universidad Autónoma de Barcelona, Bellaterra (Barcelona) España

José Tejada Fernández https://orcid.org/0000-0001-9044-8826

Director del Grupo CIFO (Colectivo de Investigación en Formación Ocupacional)

Departamento Pedagogía Aplicada, Universidad Autónoma de Barcelona, Bellaterra (Barcelona) España

$\nabla$

Recibido: 11-03-18 Revisado: 15-06-18 Aceptado: 09-10-18 Publicado: 20-12-18

- Resumen. Partiendo de un modelo previo de competencia digital docente en educación superior, se realiza un estudio mixto secuencial (QUAN+QUAL). Se parte de un diseño multietápico en el estudio de detección de necesidades de acuerdo a dos de los objetivos del estudio: identificar las competencias actuales y establecer-priorizar las necesidades formativas. La población del estudio está referida al profesorado de instituciones universitarias de la Zona Metropolitana del Valle de México. Participa una muestra de 20 instituciones (11 públicas, 9 privadas), con 247 profesores implicados. Los resultados indican un dominio medio-bajo en las competencias digitales que corresponden con el rol de docencia (planificación, el desarrollo y conducción de experiencias de aprendizaje y la evaluación con apoyo de las TIC). Seguidamente se encuentran las competencias digitales
Palabras clave: Competencia digital docente, profesor universitario, formación continua, necesidades formativas, modelo 
relacionadas con la investigación y el desarrollo profesional con apoyo de las TIC. Las competencias digitales que más domina el profesorado universitario mexicano son las que están vinculadas al compromiso y la responsabilidad social de los docentes con el uso de las TIC. Se concluye sobre las necesidades de formación más prioritarias en consonancia con el nivel medio bajo de dominio competencial verificado.

- Abstract. Based on a previous model of faculty digital competence in higher education, a mixed sequential study (QUAN+QUAL) was carried out. A multi-stage design is applied to a study focused on detecting needs according to two of the study's objectives: to identify current competences and to establish-prioritize training needs. The population of the study refers to the faculty of university institutions in the Metropolitan Area of Valle de México. A sample of 20 institutions (11 public, 9 private) participates, with 247 professors involved. The results point out to a mid-low mastery of the digital competences corresponding to the teaching role (planning, development, and delivery of learning experiences, and evaluation with the support of ICTs). Next are the digital competences related to research and professional development supported on the ICTs. The digital competences in which the Mexican university faculty is most proficient are those that are linked to the commitment and social responsibility of professors with the use of ICTs. It is concluded that there are priority training needs in line with the mid to low level of mastery of competences.

- Resumo. Partindo de um modelo prévio de competência digital docente em educação superior, realiza-se um estudo misto sequencial (QUAN+QUAL). Parte-se de um desenho multietápico para a detecção de necessidades de acordo com dois dos objetivos do estudo: identificar as competências atuais e estabelecer-priorizar as necessidades formativas. A população do estudo está referida ao corpo de professores de instituições universitárias da Zona Metropolitana do Valle de México. Consiste em uma amostra de 20 instituições (11 públicas, 9 privadas) que envolve 247 professores. Os resultados indicam um domínio médio-baixo das competências digitais condizentes com a função do professor (planejamento, desenvolvimento e condução de experiências de aprendizagem e avaliação com apoio das TIC). Seguidamente, abordam-se as competências digitais relacionadas com a pesquisa e o desenvolvimento profissional com apoio das TIC. As competências digitais que os professores universitários mexicanos dominam mais são aquelas vinculadas com o compromisso e à responsabilidade social no uso das TIC. Conclui-se sobre as necessidades prioritárias de formação cona competência que registra um domínio médio-baixo por parte dos docentes. competencia digital docente.

Keywords: faculty digital competence, university professor, continuous education, training needs, faculty digital competence model

Palavras-chave: Competência digital docente, professor universitário, formação contínua, necessidades formativas, modelo competência digital docente. 
a preocupación por la estructura competencial no es nueva, aunque sí reciente en educación superior. Ha sido a partir de la última década y en particular del 2008 (ISTE, 2008, aunque tiene orígenes anteriores; Unesco, 2008), como primeros exponentes de la emergencia de esfuerzos para examinar la competencia TIC de los profesores, produciendo otros tantos estándares sobre ella. Si bien no existe un consenso sobre el particular, nos parece de relevancia la preocupación por el perfilamiento digital docente, clave y referencia de una formación con sentido, superadora de determinados cursos de divulgación o alfabetización tecnológica más que pedagógica, sobre todo en educación superior.

La importancia que están adquiriendo las TIC para la creación de nuevos escenarios hace que la "competencia digital" sea necesaria para que la ciudadanía pueda desenvolverse en la sociedad actual y futura. Tal competencia digital debe entenderse no como el simple dominio instrumental de las mismas, sino para la construcción, producción, evaluación y selección de mensajes mediáticos.

Indudablemente que la tecnología es un factor clave, tanto para la configuración de los escenarios profesionales, como en la transformación que conllevan en la actuación profesional (Adams Becker, Cummins, Davis, Freeman, Hall Giesinger \& Ananthanarayanan, 2017; Barroso, Cabero \& Vázquez, 2012; Cabero, 2009 y 2013; Cabero \& Marín, 2012; Castañeda \& Adell, 2013; Cebrián, 2013; Del Moral \& Villaustre, 2012; Fandos, 2013; González Soto, 2009; Johnson, Adams Becker, Estrada \& Freeman, 2015; Urbina \& Salinas, 2014), en nuestro caso de los docentes, dando lugar a la modificación de las competencias existentes y a nuevos roles y nuevas competencias profesionales.

Sin lugar a dudas, las competencias digitales han cobrado un fuerte protagonismo en los últimos tiempos en los distintos niveles educativos (Almerich, Suárez, Jornet, \& Orellana, 2011; Álvarez Rojo, et al., 2009; Cabero, 2014; Carrera \& Coiduras, 2012; Domínguez, Bárcenas, RuizVelasco, \&. Tolosa, 2014; Fernández de la Iglesia, 2013; Ferrari, 2012; Gallego, Gámiz \& Gutiérrez, 2010; Gutiérrez, 2014; Mayorga \& Núñez, 2011; Medina-Talavera, 2014; Mengual \& Roig, 2012; Prendes, Castañeda \& Gutiérrez, 2010; Prendes \& Gutiérrez, 2013; Ramírez, 2012; Roig \& Pascual, 2012; Suárez-Rodríguez, Almerich, Gargallo \& Aliaga, 2013; Tejedor \& García-Valcárcel, 2006), hasta el punto de que se ha considerado como una de las ocho competencias clave para el aprendizaje permanente por parte del Parlamento Europeo (2006).

La competencia digital no solo proporciona la capacidad de aprovechar la riqueza de las nuevas posibilidades asociadas con las tecnologías digitales y los retos que plantean, sino que resulta cada vez más necesaria para poder participar de forma sistemática en la nueva sociedad y economía del conocimiento del siglo XXI (INTEF, 2013, p. 3).

Es en este contexto donde hemos centrado los objetivos de nuestro estudio: fundamentalmente en analizar e identificar el perfil y los ámbitos competenciales del profesor universitario, construir un modelo de integración de la Competencia Digital en el desarrollo 
profesional docente, de tal forma que sirva como guía y posterior punto de referencia en la autoevaluación diagnóstica de necesidades de formación continua sobre dicha competencia, a la vez que a partir del modelo propuesto y el referencial de competencias elaborado, realizar una detección de las necesidades de formación continua del profesor universitario con respecto a su Competencia Digital.

\section{Competencias digitales docentes: hacia un modelo para la integración de la competencia digital en el desarrollo profesional del docente universitario}

La competencia digital docente, bajo nuestro referente conceptual de competencia profesional, es deudora del contexto socioprofesional, junto con la acción en el sentido de resolución de problemas profesionales. Así pues, no basta con disponer de los recursos (saberes), sino que es necesario resolver problemas de manera eficaz en contextos socioprofesionales para ser competente. Este posicionamiento deviene de nuestra propia concepción de competencia en su doble dimensión: social y personal (Tejada, 2009, 2012, 2013). En el primer caso, la dimensión social, dentro de los escenarios profesionales, aludimos a las "incumbencias" en clave de funciones que se desarrollan y le son propias a un profesional específico; en el segundo caso, la dimensión personal de la competencia, nos remite al conjunto de saberes (recursos personales) para actuar competentemente.

Queremos significar en este momento la importancia del escenario, por cuanto el mismo queda condicionado por una tecnología, una organización y una normativa, que va a circunscribir de manera significativa las funciones por acometer y los recursos para activar. Nadie discute hoy la relevancia de estos tres constituyentes escénicos y la necesidad de su consideración a la hora de articular no solo cualquier perfil, sino lo más genuino para los propios propósitos de la formación de los profesionales de referencia, cual es la fuente de selección de contenidos (conceptuales, procedimentales y actitudinales). No hay que olvidar tampoco que dichos constituyentes están sujetos a cambios y en continua evolución, con lo cual tienen una fuerte presencialidad en todo lo relativo a la formación continua de los profesionales, puesto que ellos son los máximos proveedores de las necesidades de actualización de la cualificación profesional.

En la concepción y elaboración del modelo se ha intentado reflejar, tanto la integración de los avances y aciertos logrados en los referentes y modelos previos de competencias digitales docentes (Adell, 2008; Ala-Mutka (2011); Gutiérrez Porlán, 2011; INTEF, 2013; ISTE, 2008; MEN, 2013; Newrly \& Veugelers, 2009; Prendes, 2010; Prendes \& Gutiérrez; 2013; Unesco, 2008), como el esfuerzo personal por el avance conceptual y reflexivo sobre las tecnologías y el profesorado universitario - reflejado en las finalidades y orientaciones del modelo-, y el avance prácticoreflejado en las concreciones del modelo y en el desarrollo de la matriz de competencias para 
cada una de las competencias-(Pozos, 2011, 2015, 2016; Tejada \& Pozos 2018).

La finalidad principal del modelo es proporcionar al profesorado universitario una herramienta para el desarrollo y crecimiento profesional sobre las competencias digitales que debe desarrollar e integrar para desenvolverse adecuadamente en cualquiera de sus roles y funciones asociados.

El modelo pretende integrar los principales roles del perfil actual del profesor universitario (Mas \& Tejada, 2013), a través de las Competencias Digitales, así como brindar un marco claro de desarrollo profesional docente que sirva como referente para orientar sus decisiones sobre formación y actualización permanentes.

Las dimensiones básicas a través de las cuales se vertebra y constituye el modelo son:

a. Las Unidades de Competencia Digital, como punto de partida y fundamento que da sentido al modelo (la profesionalidad del docente universitario; el compromiso profesional, el impacto personal y social).

b. Las Fases de Integración de la Competencia Digital que dan el carácter de desarrollo y continuidad en el tiempo (sentido formativo).

c. El Nivel de Dominio y Grado de Complejidad de la Competencia Digital, que da cuenta del carácter evolutivo y de profundidad por niveles de desarrollo. Este aspecto será de gran utilidad en los estudios de detección de necesidades de formación continua sobre la Competencia Digital del Profesorado y su posterior priorización para conformar o planear propuestas de formación.

Sehan identificado toda una serie de Competencias Digitales para dicho perfil, quecreemos debe asumir el profesorado, haciendo énfasis en que tal competencia ha de estar integrada en el tejido mismo de cada uno de esos roles, dado su carácter transversal e impacto, en mayor o menor grado, en cada una de las funciones y actividades del docente, lo que le permitirá dejar a un lado el enfoque meramente instrumental de las tecnologías, que consideramos que es solo una parte del enfoque de competencias y que se considerará igualmente junto con aquellas competencias metodológicas, personales y participativas que conforman todos los tipos de saber de una competencia (Tejada, 2007) y abrirá paso a la prioridad de la persona, del profesional, para que sea él quien a partir de la reflexión crítica, utilice e integre adecuadamente las TIC no solo en el aula, que correspondería sólo al rol de docencia, sino que las integre en cada uno de sus roles y funciones profesionales y sus contextos correspondientes, de acuerdo a su criterio y experiencia profesional. Así, el enfoque no estará en la tecnología, sino en el poder de reflexión y decisión del ser humano para utilizar estratégicamente dicha tecnología.

La opción por un modelo de competencias digitales a partir de las funciones y roles deviene de nuestro propio planteamiento escenario profesional (contexto) y las funciones que son de su incumbencia en el mismo (Pozos, 2011, 2013; Tejada, 2009).

Por tanto, en congruencia con las teorías sobre el desarrollo profesional docente, se 
ha definido que la integración de la Competencia Digital del profesor ha de estructurarse en tres grandes fases (competencias básicas, de profundización y de generación del conocimiento) (UNESCO, 2008, 2011), concebidas como un proceso complejo en el que el profesorado va profundizando en el conocimiento y uso de las TIC y las va integrando gradual y significativamente en su actividad docente a través de la reflexión crítica, como elemento esencial en todo este proceso.

Concebir la integración de la competencia digital en el desarrollo profesional docente como proceso continuo, recurrente y gradual (aprendizaje permanente), implica, además, definir el grado o medida en que cada una de las competencias puede irse desarrollando y profundizando, tanto en su comprensión como en su utilización e integración significativa, es decir, el grado de dominio o maestría de la competencia digital. Así, hemos definido cinco niveles que inician desde la consideración que una competencia no ha sido desarrollada hasta el momento, pero que sin embargo está latente por aprender y desarrollar, hasta llegar a un ideal o máximo nivel de desarrollo de la competencia, como experto.

En el Apéndice A ofrecemos una matriz del despliegue de las competencias con sus descriptores.

\section{MÉTODO}

\section{Diseño}

Se ha realizado un estudio descriptivo exploratorio mixto, de tipo transformador-concurrente (QUA+QUAL), en el que a ambos enfoques metodológicos se les brinda la misma relevancia y la recopilación de datos es simultánea (Hernández Sampieri, Fernández Collado \& Baptista 2014). Se parte de un diseño multietápico en el estudio de detección de necesidades en el marco de la elaboración de planes y programas formativos (Tejada \& Giménez, 2007), en consideración con uno de los objetivos de la investigación (identificar y analizar las competencias actuales) en el contexto del estudio de campo -competencias actuales- y establecer-priorizar las necesidades -necesidades formativas.

\section{Participantes}

La población del estudio está referida al profesorado deInstituciones deEducación Superior dela Zona Metropolitana del Valle de México. Participa una muestra de 20 instituciones (11 públicas, 9 privadas), con 247 profesores implicados. La caracterización de la muestra del profesorado se concreta en torno a una edad media de 45,47 años; 49\% masculina y 51\% femenina; de campos de conocimiento de ciencias sociales (30\%), ciencias naturales, exactas y computación (24\%); artes y humanidades (18\%); y 15 \% de ciencias de la educación; mayoritariamente a tiempo completo (55\%), con formación continua en áreas relacionadas con la educación y la tecnología en el 
76\%; provenientes en su mayor parte $(72,47 \%)$ de las universidades privadas y en menor medida (27,53\%), de las universidades públicas.

\section{Instrumentos de recolección de datos}

Dada la naturaleza del objeto de estudio, se optó por diversas técnicas e instrumentos de investigación, tanto cuantitativos como cualitativos, desde una perspectiva de triangulación para dotar de veracidad o autenticidad a la información recogida (Tójar, 2006). En el proceso de triangulación se utilizaron diferentes perspectivas y fuentes de indagación (profesorado, alumnado, colegas, directivos, investigador) que se combinaron con diversas técnicas e instrumentos de investigación (cuestionarios -247-, entrevistas en profundidad -30- y grupos de discusión -4-).

Para el diseño de cada una de las técnicas e instrumentos del dispositivo de recogida de información, se partió de un profundo proceso de reflexión y análisis del estado de la cuestión a nivel nacional e internacional, para poder configurar la base teórica-metodológica de la conceptualización y construcción, tanto del modelo de integración de la competencia digital en el desarrollo profesional docente, punto central en la presente investigación, como de la matriz de competencias digitales derivada del modelo.

En este documento solo abordaremos lo relativo al cuestionario, desde su construcción validación, así como los resultados obtenidos desde la perspectiva del nivel de dominio y necesidad de competencia digital docente.

La matriz quedó conformada finalmente por 78 unidades de competencias organizadas en las 7 competencias digitales del modelo y en los 3 niveles de profundización desarrollados en el mismo; a partir de dichas competencias se construyó todo el dispositivo de recogida de datos de la investigación (Apéndice A).

La escala de medida numérico-descriptiva ha sido doble, en consideración al nivel de dominio actual de la competencia (1=no desarrollada, 2=dominio básico, 3=dominio medio, 4=dominio alto, 5=dominio total), así como a la necesidad de dominio de la misma (1=ninguna, 2=poca, 3=regular, 4=alta, 5=imprescindible).

\section{Procedimiento}

La elaboración del cuestionario partió de la matriz de competencias derivadas del modelo propuesto y se siguieron los pasos habituales en su diseño (preparación, primera elaboración, validación por expertos y aplicación piloto y elaboración definitiva (Mateo \& Martínez, 2008). En nuestro caso, dos de los procesos clave en la elaboración fueron, por un lado, la validación del cuestionario a través de jueces expertos, y por otro, la aplicación de una prueba piloto en el contexto universitario mexicano. Para ambos procesos, se llevaron a cabo los análisis estadísticos y las pruebas pertinentes, que nos permitieron obtener evidencias significativas 


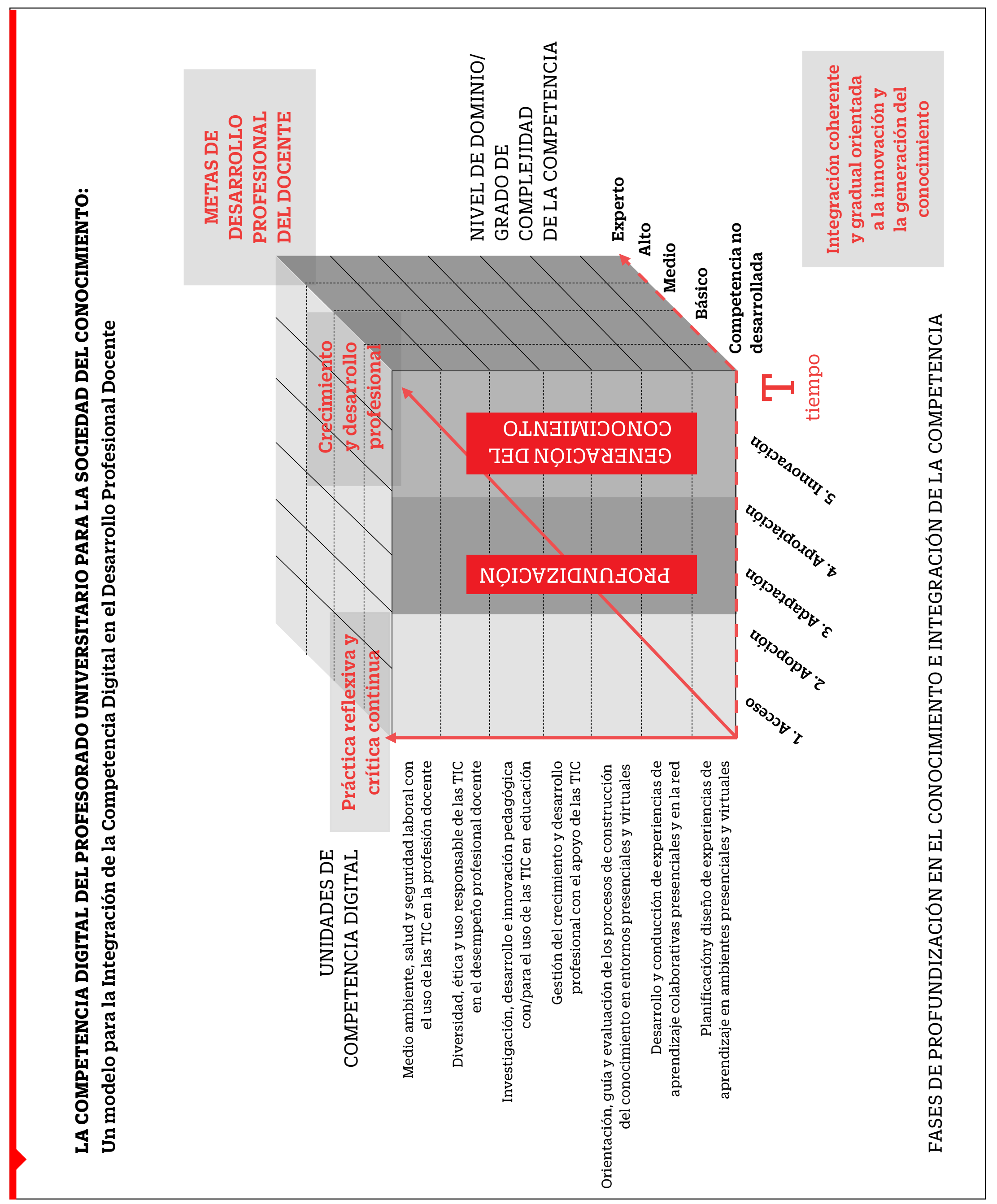

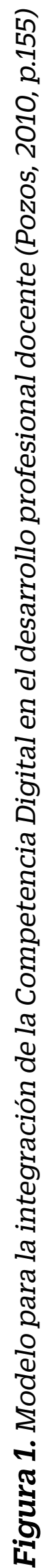


acerca de la validez y la alta confiabilidad del instrumento (alpha de Cronbach $=0.989$ ), concluyendo que es un instrumento fiable, con una escala de medida que recoge mediciones estables y consistentes (Pozos, 2013).

El tratamiento estadístico de los datos obtenidos a través del cuestionario fue llevado a cabo con el programa estadístico SPSS v19.0, la graficación para el análisis de las discrepancias encontradas en las competencias digitales fue realizada en Excel v2007, a partir de las medias y desviaciones estándar obtenidas.

\section{RESULTADOS}

Como ya hemos apuntado, en este documento, solo presentamos los resultados relativos al cuestionario y más concretamente los ítems específicos de las unidades de competencia digitales docentes consideradas en nuestro modelo.

Los resultados están organizados en dos grandes bloques: uno, para presentar el análisis de los datos específicamente sobre el dominio y necesidad en competencia digital, y un segundo, para el análisis inferencial de estos datos.

Para identificar si existía una discrepancia o necesidad, se consideró que la media de la Necesidad tendría que ser más alta estadísticamente que la media del Dominio (prueba t de Student para muestras relacionadas).

Cuando sí hay Necesidad Formativa, hay que valorar si ésta es significativa, es decir, identificar y priorizar cada una de ellas para orientar la toma de decisiones formativas. De acuerdo a la misma escala del cuestionario, cuando hay necesidad establecimos los siguientes criterios:

Tabla 1

Criterios de decisión para la formación

\begin{tabular}{|c|c|c|}
\hline CRITERIO (puntos escala) & VALORACIÓN & DECISIÓN PARA LA FORMACIÓN \\
\hline $\begin{array}{l}(1,2) \\
(3,5)\end{array}$ & $\begin{array}{l}\text { Dominios de nivel bajo } \\
\text { Necesidades altas }\end{array}$ & = PRIORIDAD ALTA \\
\hline $\begin{array}{c}(1,2) \\
(2,2.99)\end{array}$ & $\begin{array}{l}\text { Dominios de nivel bajo } \\
\text { Necesidades medias }\end{array}$ & = PRIORIDAD MEDIA \\
\hline $\begin{array}{c}(2,1,3) \\
(3,5)\end{array}$ & $\begin{array}{l}\text { Dominios medios } \\
\text { Necesidades medias }\end{array}$ & = PRIORIDAD BAJA \\
\hline
\end{tabular}




\section{Análisis del DOMINIO ACTUAL vs NECESIDAD}

En el gráfico siguiente, se presenta un panorama global del dominio actual en comparación a las necesidades expresadas en la UC1, PLANIFICACIÓN Y DISEÑO DE EXPERIENCIAS DE APRENDIZAJE, integrando todas las unidades en sus tres niveles (Básicas, Profundización y Generación del Conocimiento).

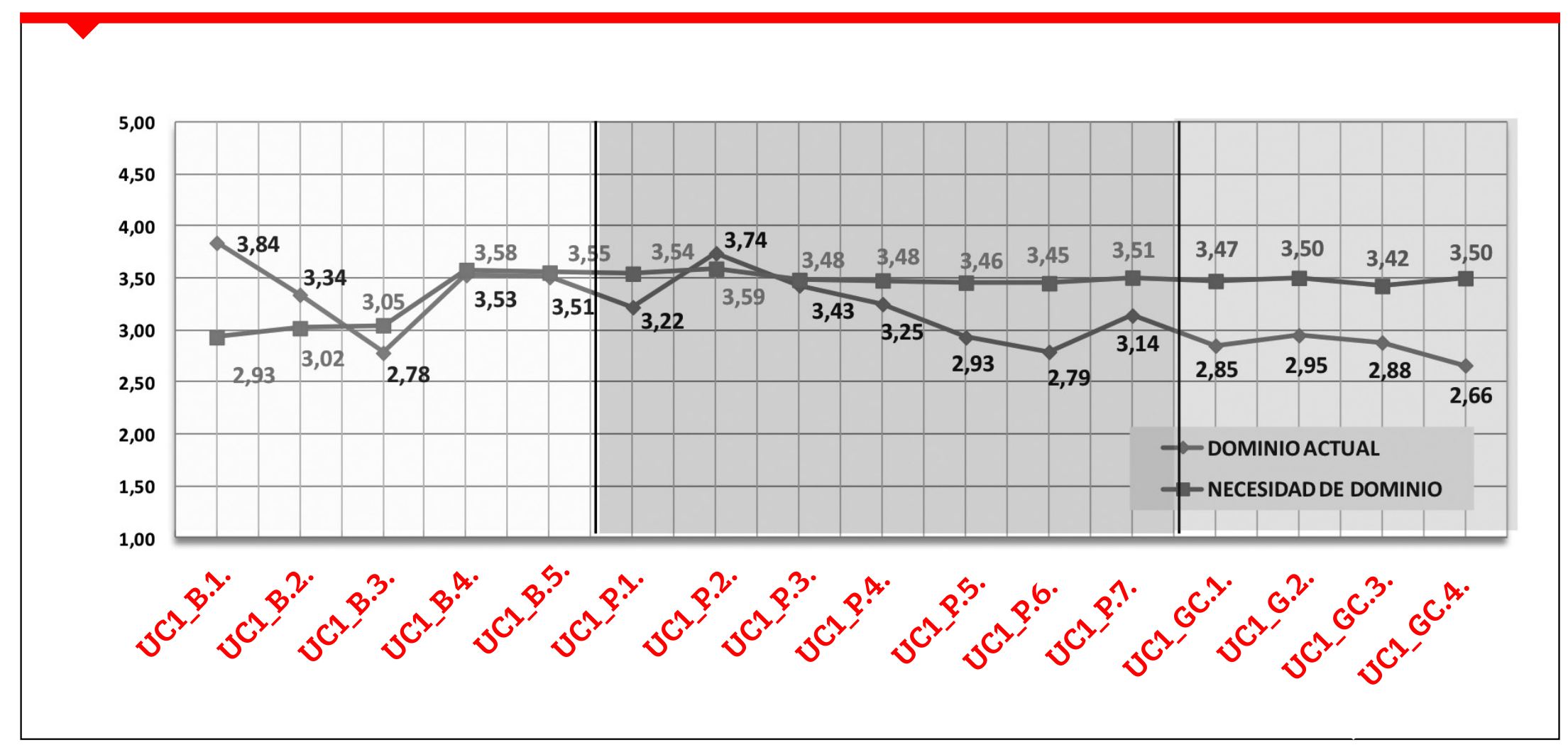

Figura 2. C1_PLANIFICACIÓN Y DISEÑO de experiencias de aprendizaje en ambientes presenciales y virtuales

Tomando como referencia la media teórica de nuestra escala $\mathrm{x}=3$ (dominio medio), nos dice que en este perfil, el 56,25\% de las unidades de esta competencia (9/16) se encuentran por arriba de la media teórica y que el $43,75 \%$ restante (7/12) se encuentra por debajo, pero muy cerca de ella (entre 2,66 y 2,95); esto significa, de acuerdo con nuestra escala, que el dominio actual de estas competencias está principalmente entre un nivel básico y un nivel medio de desarrollo, conllevando necesidades formativas relacionadas con los niveles de profundización y generación del conocimiento.

Observamos que en este perfil de la UC2 DESARROLLO Y CONDUCCIÓN DE EXPERIENCIAS DE APRENDIZAJE COLABORATIVOS, el 58\% de las competencias digitales de los profesores (7/12) se encuentran por debajo de la media teórica, lo que significa, de acuerdo con nuestra escala, que el profesorado mexicano domina actualmente estas competencias en un nivel básico. El 42 $\%$ de competencias digitales restante en esta competencia (5/12), tienen medias superiores a la media teórica, por tanto, representa que el profesorado tiene un dominio actual de nivel medio. 


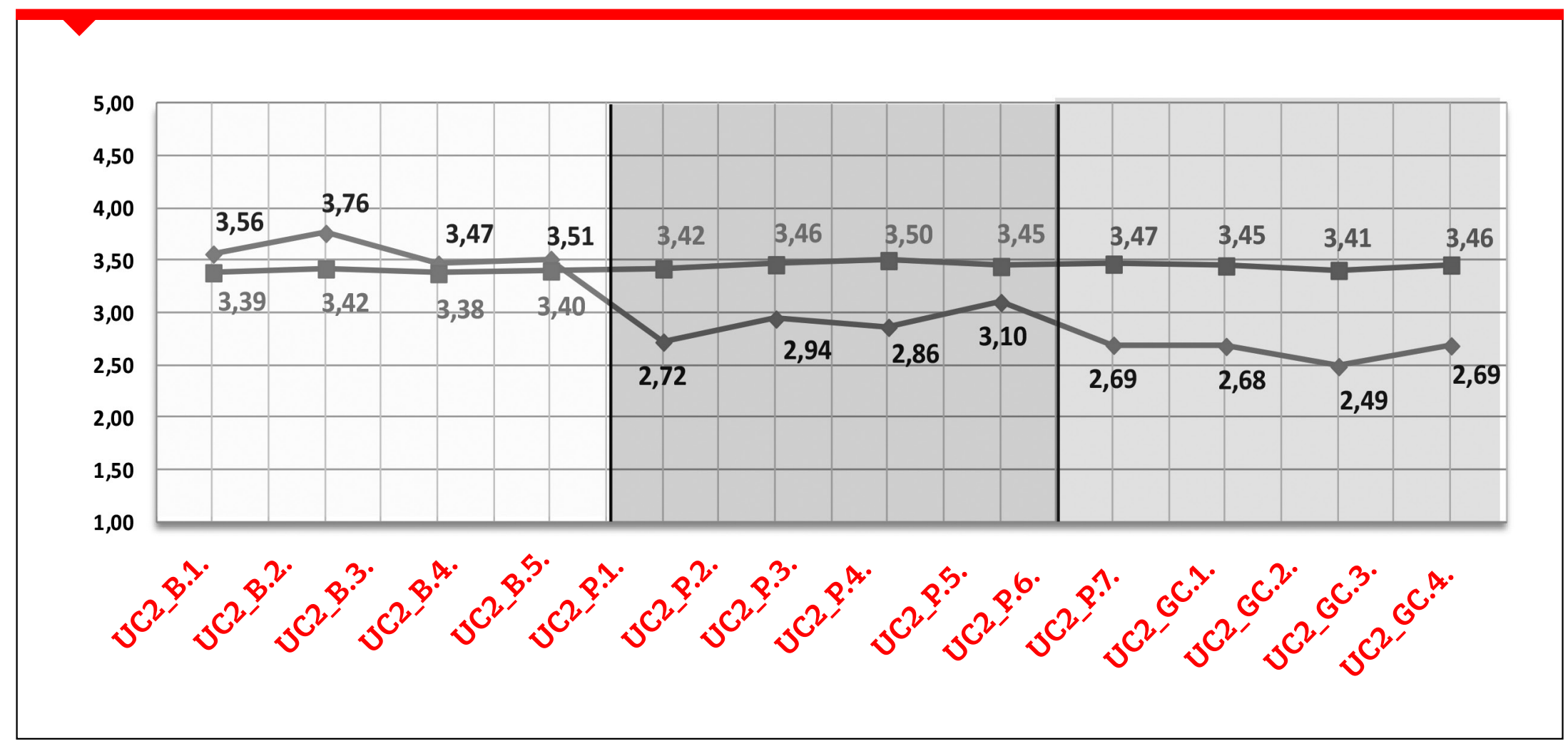

Figura 3. C2_Global. DESARROLLO Y CONDUCCIÓN de experiencias de aprendizaje colaborativas presenciales y en red

UC3_GLOBAL: Competencias Digitales para la orientación, guía y evaluación de los procesos de construcción del conocimiento de los alumnos en entornos presenciales y/o virtuales

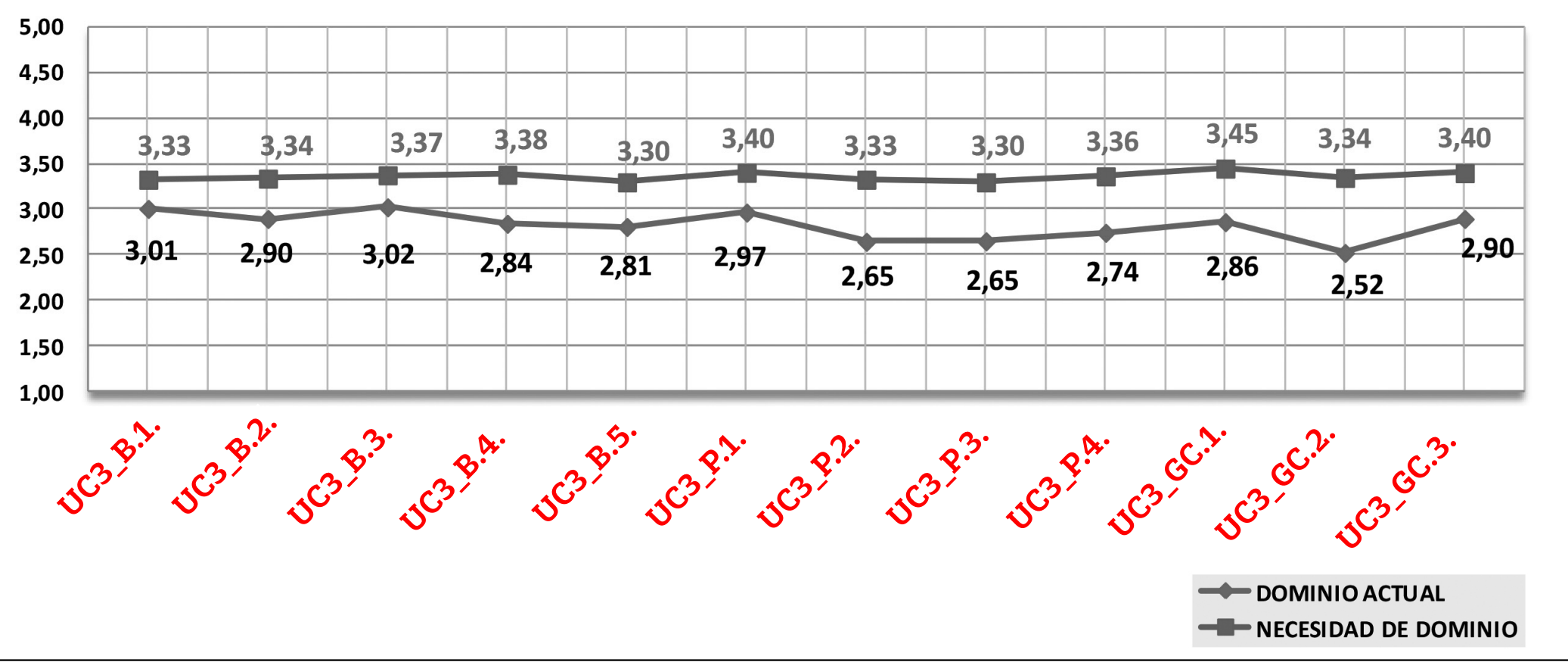

Figura 4. C3_Global. ORIENTACION, GUIA Y EVALUACION de los procesos de construcción de los alumnos en entornos presenciales $\mathrm{y} / \mathrm{o}$ virtuales

En suma, el dominio actual de estas competencias está principalmente entre un nivel básico y medio de desarrollo, de lo que se deriva igualmente que en la mayor parte de las unidades (8/12) se presentan necesidades formativas. 
En el caso de la Competencia 3. ORIENTACIÓN, GUIA Y EVALUACION DE LOS PROCESOS DE CONSTRUCCIÓN DEL CONOCIMIENTO DE LOS ALUMNOS, tomando nuevamente como referencia la media teórica, vemos que casi todas las unidades de competencia se sitúan por debajo de la media, es decir, que el 83.3\% de estas competencias digitales (10/12) presentan un dominio actual básico o poco desarrollado por parte del profesorado. El 16.7\% restante de competencias digitales (2/12), apenas alcanzan la media (UC3_B.1, UC3_B.3) y, por tanto, las dominan en un nivel medio.

Se presenta nuevamente el panorama global de esta competencia donde se observan necesidades formativas que gradualmente se hacen un poco más amplias en el nivel de Generación del conocimiento.

Siguiendo el mismo procedimiento anterior para la C4. GESTIÓN DEL CRECIMIENTO PROFESIONAL CON APOYO DE LAS TIC, observamos que en este perfil, el 70\% de las competencias digitales de los profesores (7/10) se encuentran por arriba de ésta [3.04, 3.97] , lo que significa que el dominio actual de estas competencias está un nivel medio tendiendo hacia un nivel alto, ya que hay también competencias que se acercan más 4 y que se tiene un dominio alto sobre ellas.

UC4_GLOBAL: Competencias Digitales para la gestión del crecimiento profesional con apoyo de las TIC

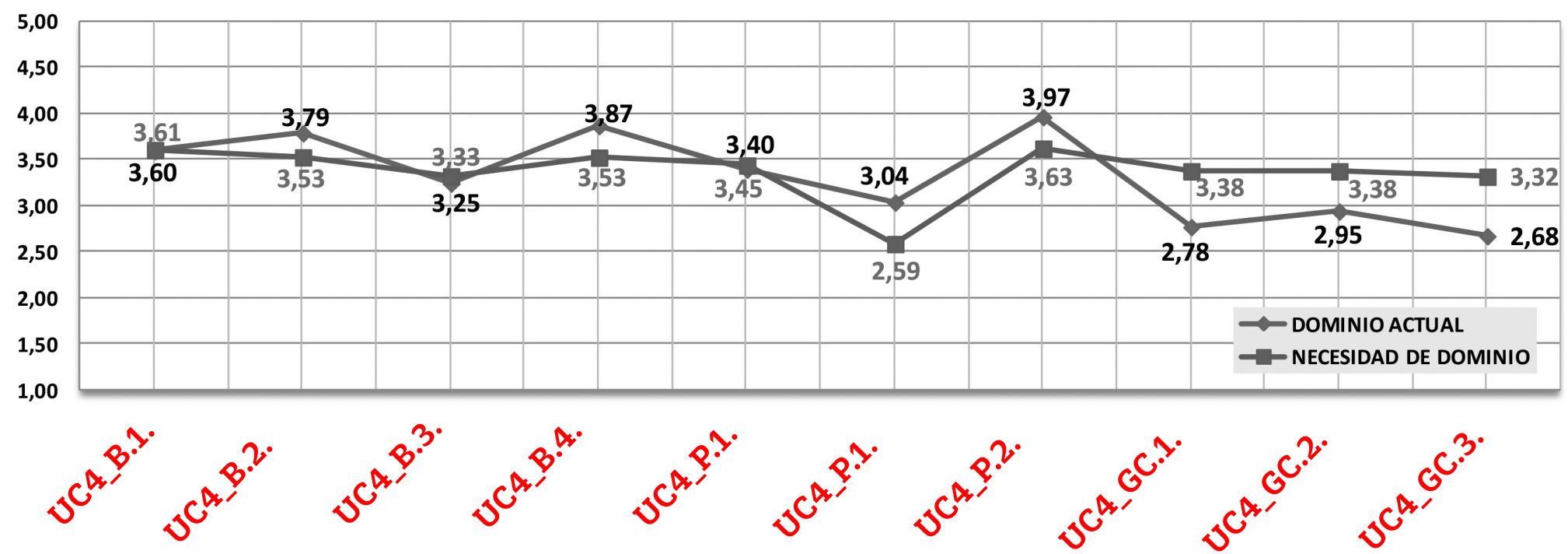

Figura 5. C4_Global. GESTION del crecimiento profesional con la ayuda de las TIC

En menor medida, el otro 30 \% de competencias restantes (3/10), se encuentran con puntuaciones bajas por debajo de la media teórica, por tanto, situándose en un dominio actual básico sobre éstas (UC4_GC.1, UC4_GC.2 y UC4_GC.3), pero tendiente hacia un nivel medio.

Como vemos, las necesidades formativas se incrementan más en las referentes a la generación del conocimiento. 
En el caso de la C5. INVESTIGACIÓN, DESARROLLO E INNOVACIÓN PEDAGÓGICA CON TIC, observamos que en este perfil, que el 60\% de las competencias digitales de los profesores (6/10) se encuentran por arriba de la media teórica, lo que significa, de acuerdo con nuestra escala, que el dominio actual de estas competencias está un nivel medio a alto, ya que además hay una competencia (UC5_B1) que tiene un valor mayor de 4 que corresponde a un dominio alto, esta se refiera a la búsqueda y consulta de información relevante a través de la red y las tecnologías para el desarrollo de actividades para la docencia, la investigación y la gestión. El $40 \%$ de competencias digitales (4/10), tienen medias inferiores a la media teórica [2.35, 2.80], por tanto, representa que el profesorado tiene un dominio actual de nivel básico o poco desarrollado sobre estas competencias (UC2_P.1, UC2_P.3, UC2_GC.2, UC2_GC.3).

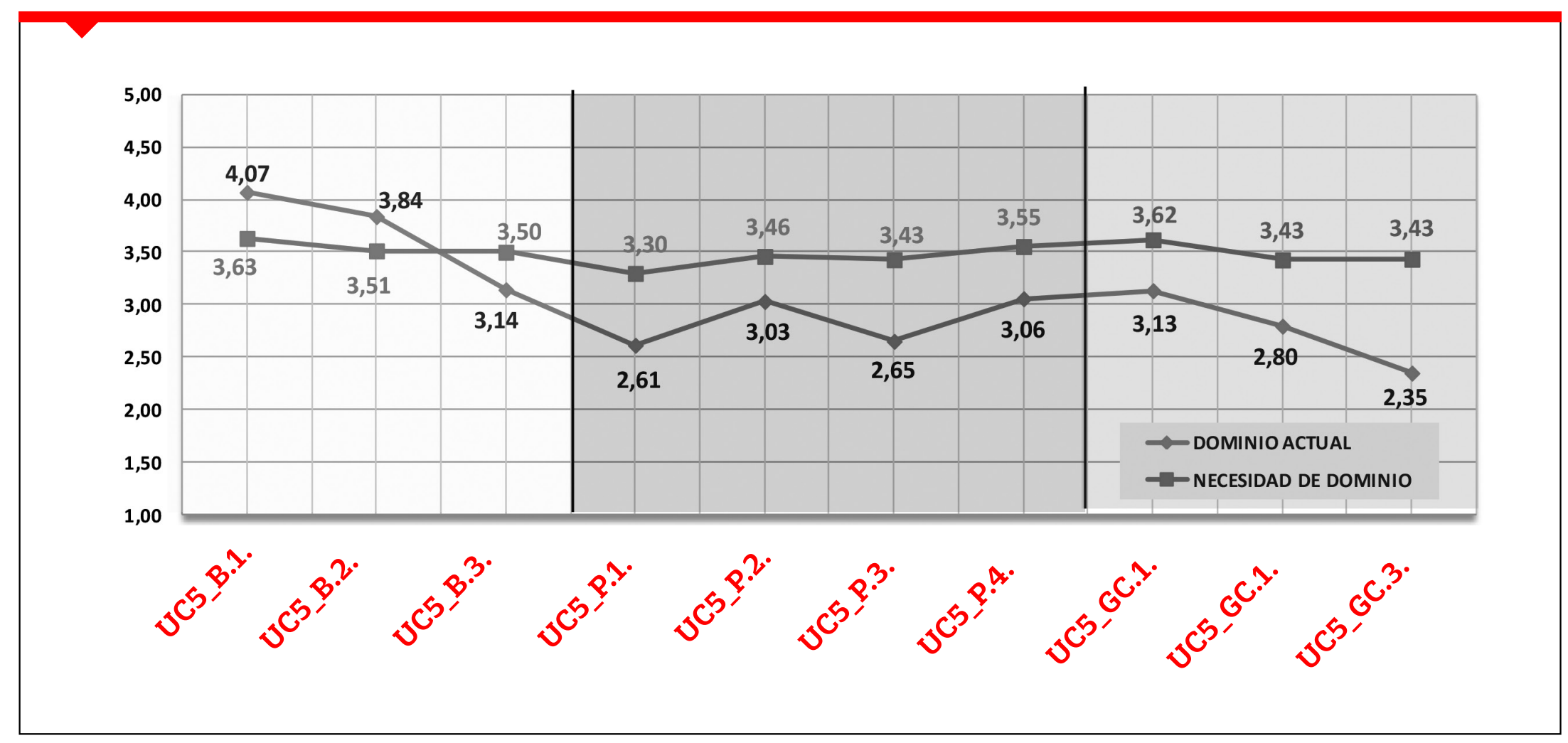

Figura 6. C5_Global. INVESTIGACIÓN, DESARROLLO e INNOVACIÓN pedagógica con/para el uso de las TIC

En la mayor parte de las unidades de competencia de esta competencia se observan necesidades formativas (8/10) dado que el nivel de necesidad expresada es mayor al nivel de dominio actual de las competencias.

El perfil que muestra la competencia C6. MANEJO DE LA DIVERSIDAD, ETICA Y USO RESPONSABLE DE LAS TIC nos indica un dominio mayor del que se ha visto en todas las competenciales anteriores, ya que no solo se observa que todas están por arriba de la media (11/11), sino que el 27\% de ellas (3/11) está dentro del nivel de domino alto (UC6_B2, UC6_P5 y UC6_GC2), y el resto en un dominio medio alto.

En este sentido, a partir del dominio actual, podemos considerar que no se consideran necesidades formativas, dado que las discrepancias vienen asociadas a que se tiene mayor dominio que necesidad, excepto en algunas de ellas. 


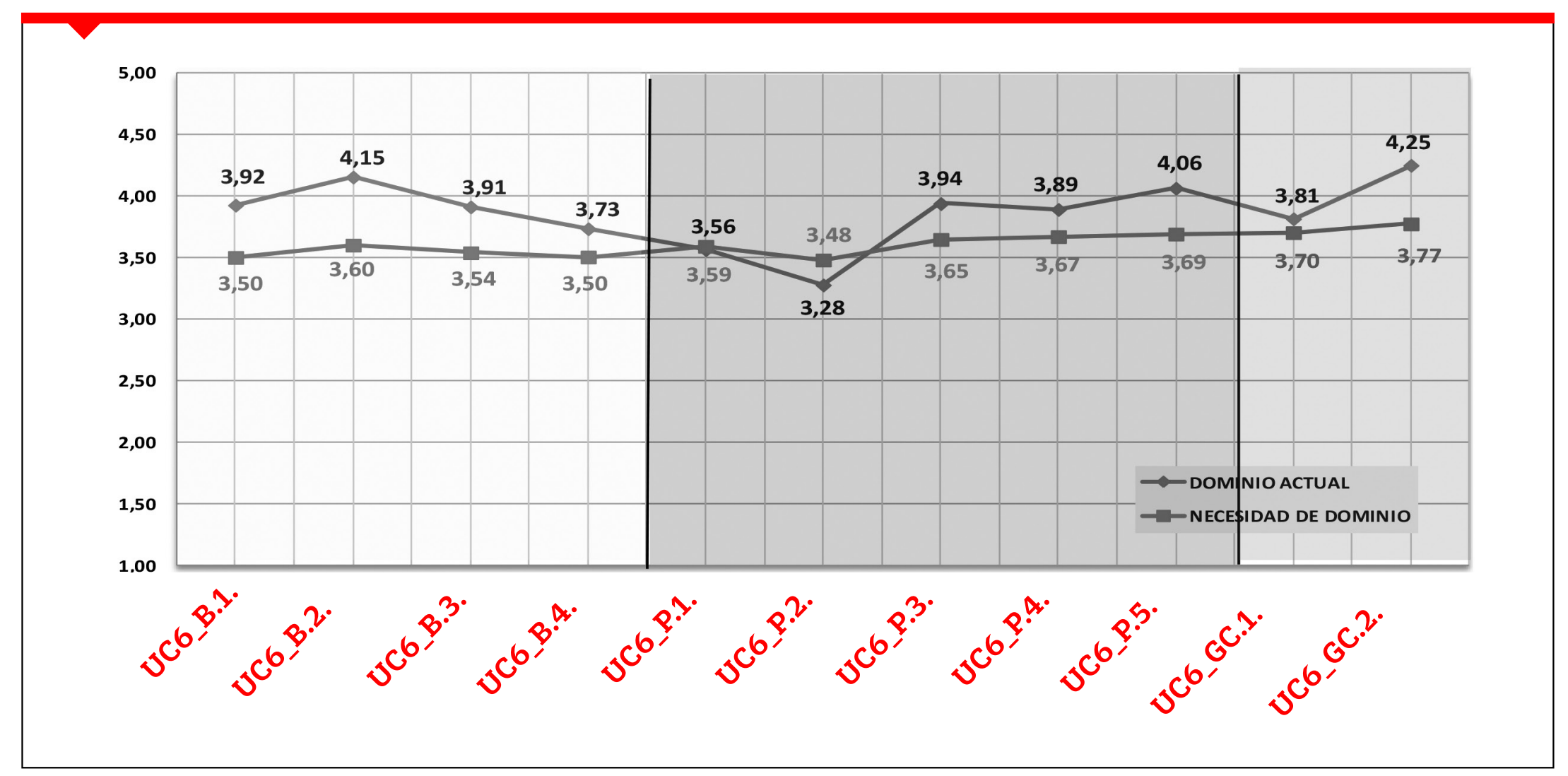

Figura 7. C6_Global. DIVERSIDAD, ÉTICA Y USO RESPONSABLE de las TIC

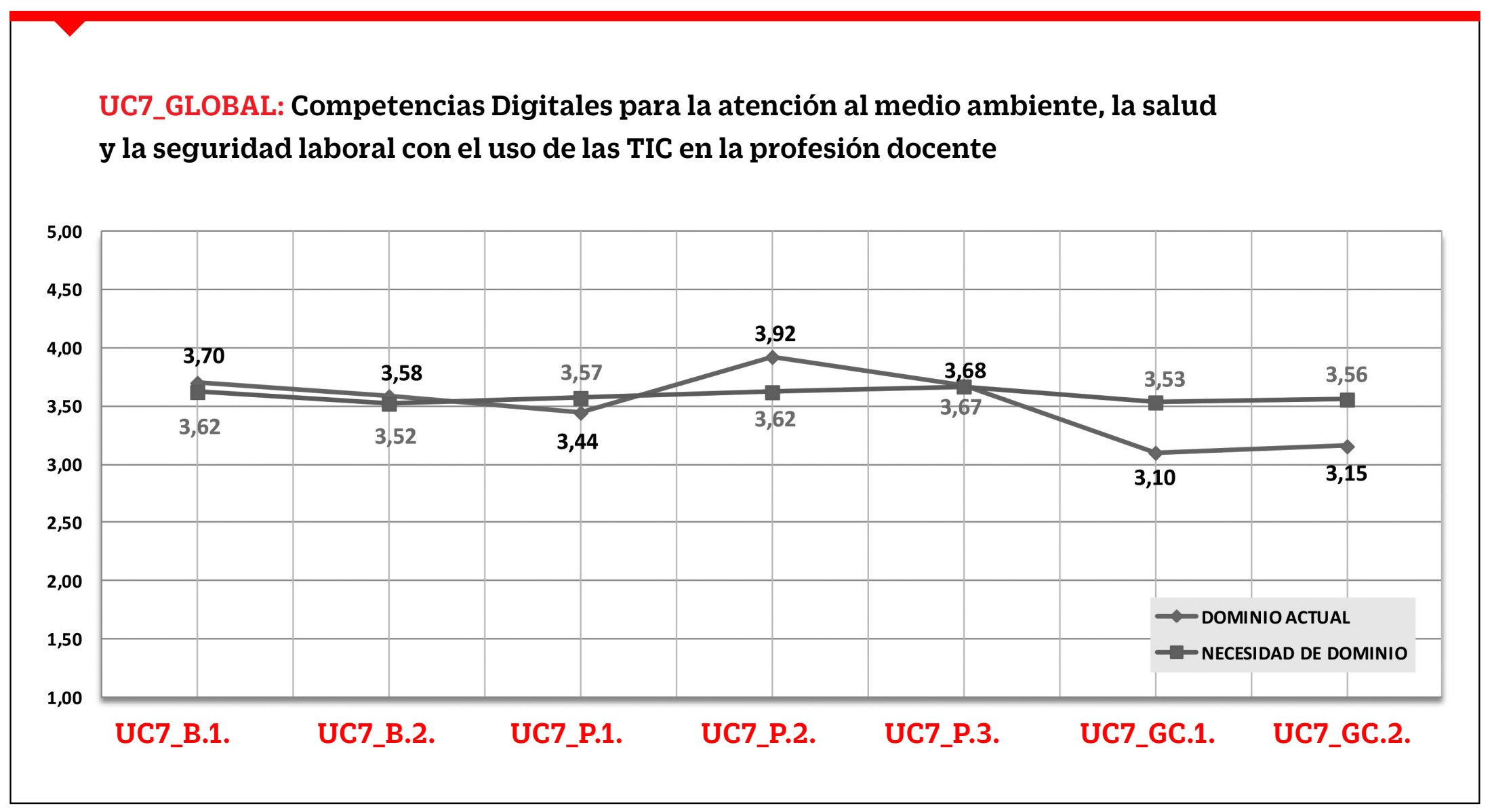

Figura 8. C7_Global. MEDIO AMBIENTE, SALUD Y SEGURIDAD LABORAL con el uso de las TIC

Por último, en la competencia C7: MEDIO AMBIENTE, SALUD Y SEGURIDAD LABORAL CON EL USO DE LAS TIC, se verifica que el 100\% de las competencias digitales de los profesores (7/7) se encuentran por encima de la media, lo que significa un dominio actual medio o de 
considerable importancia de estas competencias. Incluso, el 57\% de ellas (UC7_B1, UC7_B2, UC7_ B1, UC7_P2 y UC7_P3), se acercan más a una puntuación de valoración más alta sobre el dominio

En suma, solo en tres unidades de competencia (UC7_P1, UC7_GC1 y UC7_GC2) se observa una discrepancia, en donde la necesidad expresada es mayor al dominio y, por tanto, solo aquí se vislumbran necesidades formativas.

\section{Análisis inferencial}

Considerando ahora de manera global las 7 competencias digitales, tanto en dominio como en necesidad, también vemos similares resultados entre hombres y mujeres, es decir, que no hay diferencias considerables por género, excepto en la competencia 7, donde las mujeres muestran nuevamente un mayor dominio que los hombres $(\mathrm{p}<0,05)$.

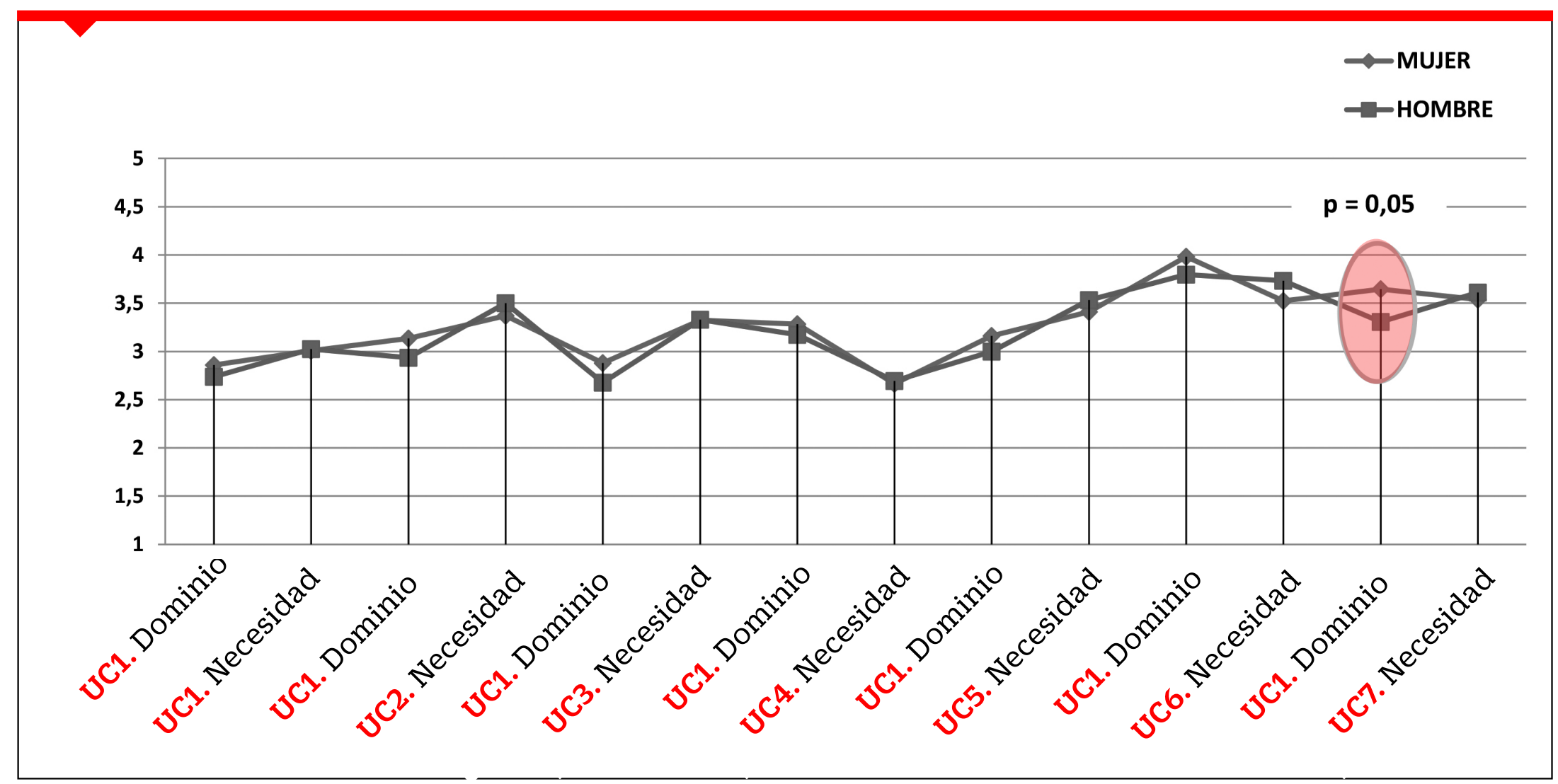

Figura 9. Diferencias entre el dominio y la necesidad por género

Si consideramos globalmente las competencias, tanto en dominio como en necesidad, también se observa similar valoración entre las instituciones públicas y las privadas, excepto en la competencia 3, donde el profesorado de las IES privadas muestra un dominio significativamente más alto que el de las IES públicas $(p<0,05)$ y que se refiere a la orientación, guía y evaluación de los procesos de construcción del conocimiento en los entornos de aprendizaje presenciales y virtuales. 


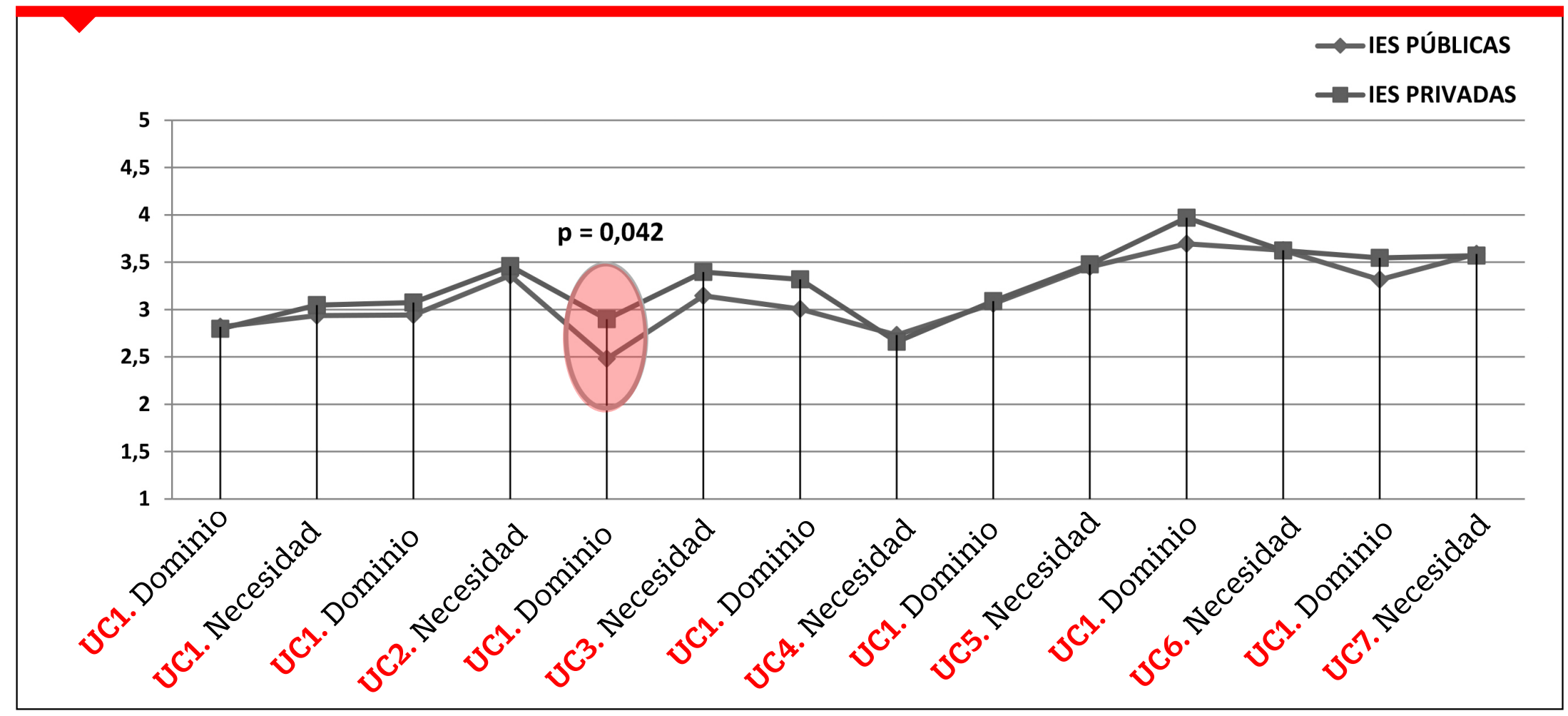

Figura 10. Diferencias entre el profesorado de IES públicas e IES privadas

Comparando nuevamenteel dominio y la necesidad del profesorado que sí tiene formación continua y el que no, observamos resultados dispares, es decir, que, con respecto a la formación continua, sí hay diferencias significativas principalmente en las competencias relacionadas con la docencia tanto presencial, virtual y mixta, como con la gestión, la investigación y la innovación pedagógica con TIC (en esta última solo en el dominio). Cabe mencionar que donde no hay diferencias significativas, es en la necesidad de la competencia 5 (que es la referida a la investigación e innovación pedagógica con TIC), y en las competencias 6 y 7, que son las que están más relacionadas con la responsabilidad social del profesorado en su profesión docente.

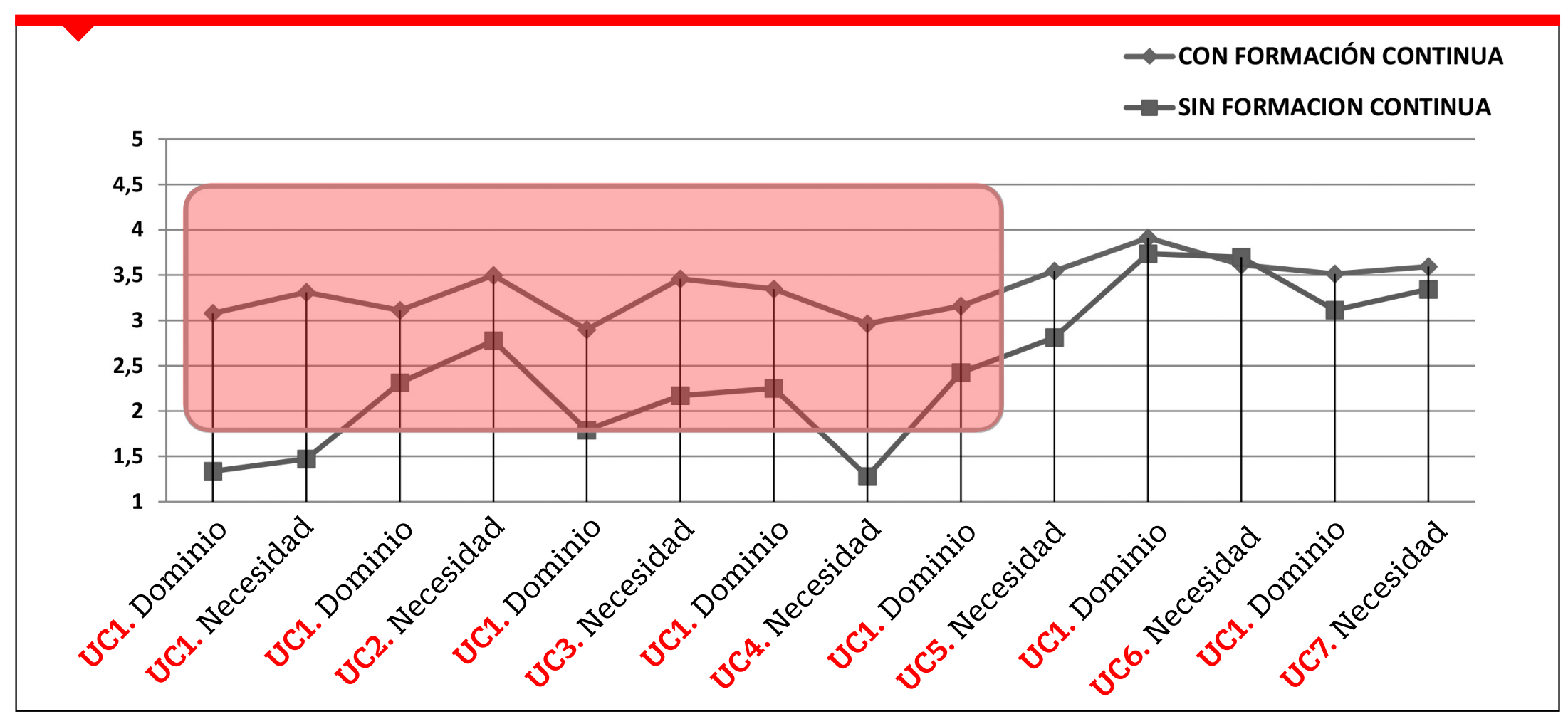

Figura 11. Diferencias entre el dominio en función de la formación continua 


\section{Discusión}

A partir de la identificación de las problemáticas de cualificación profesional en el docente universitario en la actualidad, se integró la variable TIC, tratando de averiguar en qué aspectos o roles, de qué forma y en qué medida, las TIC han impactado en el perfil del profesorado, sus funciones y contextos de actuación. Identificamos que el Profesor Universitario ha de desarrollar siete competencias digitales para la Sociedad del Conocimiento:

1) Planificación y diseño de experiencias de aprendizaje en ambientes presenciales y virtuales.

2) Desarrollo y conducción de experiencias de aprendizaje colaborativas presenciales y en red.

3) Orientación, guía y evaluación de los procesos de construcción del conocimiento en entornos presenciales y virtuales.

4) Gestión del crecimiento y desarrollo profesional con apoyo de las TIC.

5) Investigación, desarrollo e innovación pedagógica con/para el uso de las TIC en educación.

6) Diversidad, ética y uso responsable de las TIC en el desempeño profesional docente.

7) Medio ambiente, salud y seguridad laboral con el uso de las TIC en la profesión docente.

La elaboración específica de cada una de las competencias se llevó a cabo principalmente teniendo en cuenta una conceptualización de las competencias, influida tanto por una perspectiva holística, integral y compleja, comola dealgunas delas reflexiones más importantes sobre las competencias docentes (Ala-Mutka, 2011; Mas \& Tejada, 2013; Navío, 2005; Prendes, 2010; Tejada, 2009; UNESCO, 2008). Con ello, se logró desarrollar un Modelo de Integración de la Competencia Digital, y una vez, establecido su dominio, identificar sus propias necesidades de formación continua con respecto a las mismas posteriormente (Pozos, 2011, 2015, 2016; Tejada \& Pozos 2018).

Como podemos observar, en el siguiente gráfico, se muestra la síntesis del dominio global de las competencias digitales del profesorado de la muestra, ordenadas de mayor a menor nivel de desarrollo, y en la que nos percatamos de que las competencias 1, 2 y 3, son las menos desarrolladas (prácticamente por debajo de la media teórica), y por lo tanto, presentan mayores necesidades formativas; estas competencias digitales se corresponden justamente al rol de docencia, y de ellas, las más débiles son las competencias digitales relacionadas con la planificación, así como el desarrollo y conducción de experiencias de aprendizaje presenciales con TIC, o bien, mixtas o totalmente virtuales, y posteriormente, las relacionadas con la evaluación con apoyo de las TIC o la evaluación y seguimiento del aprendizaje en ambientes virtuales. Seguidamente, y por encima de la media teórica, pero sin llegar a un nivel considerablemente avanzado, se encuentran las competencias digitales relacionadas con la investigación y el desarrollo profesional con apoyo de las TIC, así como las vinculadas a la 
gestión del crecimiento y desarrollo profesional apoyados por las tecnologías. En este grupo de competencias el profesorado tiene un nivel de dominio medio o regular, que sobrepasa ligeramente por arriba de la media teórica.

Las competencias digitales que más domina el profesorado universitario mexicano estudiado son las que están vinculadas al compromiso y la responsabilidad social de los docentes con el uso de las TIC, como por ejemplo, el desarrollar y brindar materiales digitales y experiencias de aprendizaje enriquecidas con TIC, considerando la diversidad lingüística y cultural de los alumnos; o bien, fomentar el uso ético, legal y responsable de las TIC en los alumnos a través del análisis crítico de las implicaciones y consecuencias de su uso inadecuado.

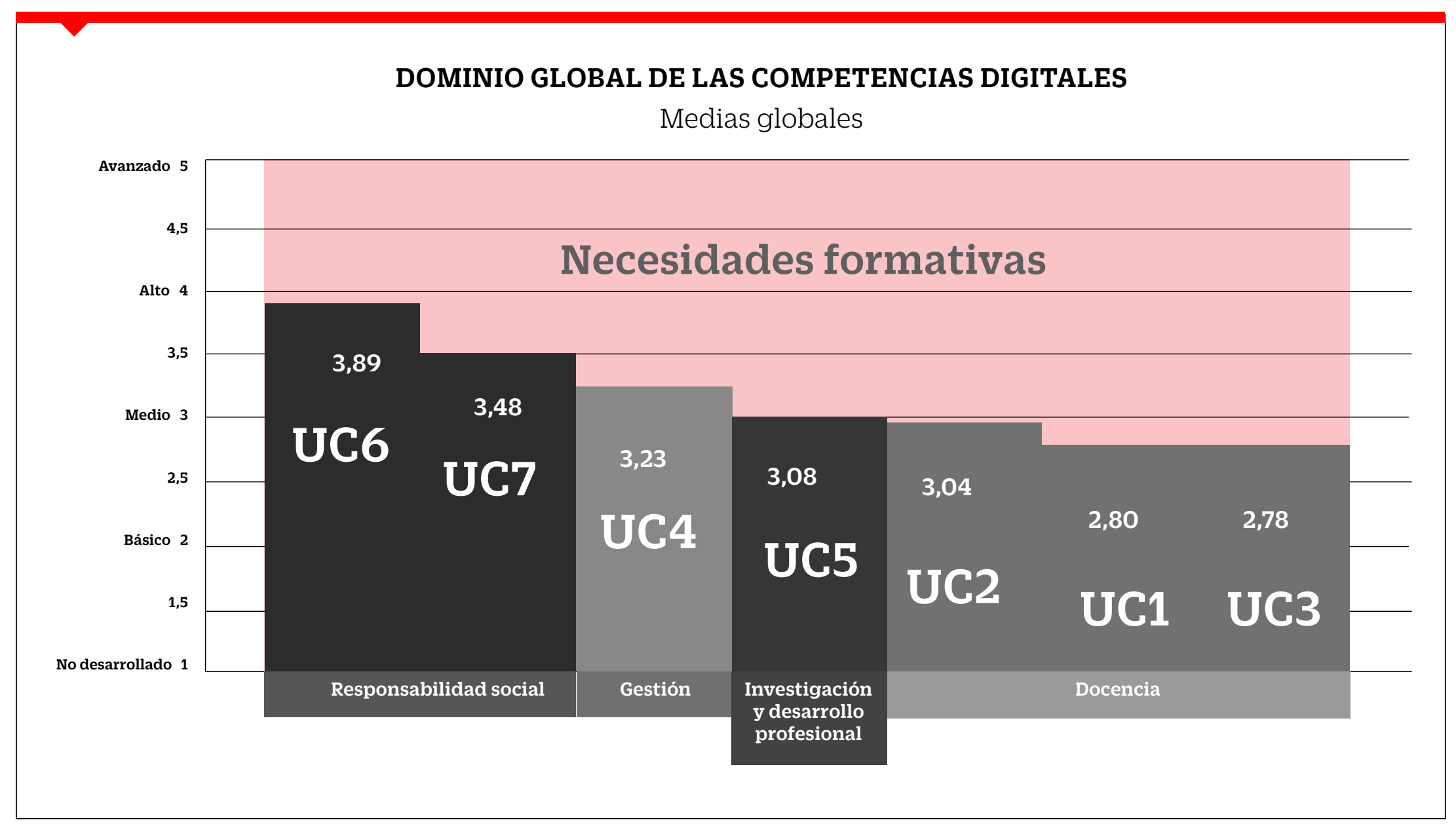

Figura 12. Niveles de dominio y necesidades formativas en competencias digitales docentes

El peso de las necesidades formativas encontradas se puede observar en el gráfico que muestra el nivel de dominio actual, y donde podemos dibujar el área que falta por desarrollar para un desarrollo óptimo de las competencias digitales. Por ejemplo, dado que las competencias digitales para la docencia fueron en las que el profesorado tiene menor dominio, es ahí donde destacamos más necesidades y, por tanto, las prioridades formativas estarían principalmente en esta área. 
Así pues, como apuntamos en el apartado de resultados, se encontraron un total de 42 necesidades formativas distribuidas en 6 de las 7 competencias; de ellas, 33 resultaron con prioridad alta y 9 con prioridad baja. Las necesidades formativas con prioridad alta corresponden básicamente a las competencias vinculadas con la docencia, la investigación y la gestión en ambientes presenciales, mixtos y virtuales; estas altas necesidades se observan especialmente en los niveles de profundización y de generación del conocimiento, por lo que corroboramos que el dominio actual del profesorado en competencia digital está, de manera general, en un nivel bajo-medio y que tiene mucha relación con lo expresado por el profesorado en las entrevistas y grupos de discusión, sobre todo al afirmar que, aun teniendo la tecnología, no le saben sacar todo el provecho que se le podría sacar para la enseñanza y el aprendizaje. Las necesidades formativas con prioridad baja corresponden mayormente a la competencia 5 que se refiere a aquellas competencias digitales necesarias para la investigación, desarrollo e innovación pedagógica con TIC y para el uso de las TIC en la enseñanza universitaria y que también se corresponde con lo apuntado por el profesorado, ya que expresa que hace falta más investigación sobre los usos y beneficios reales de las tecnologías en el aprendizaje.

Con todo, también hemos de concluir que lo anterior es extensivo tanto a profesores como profesoras, y a instituciones públicas y privadas en igual medida. Si bien, hemos verificado que el nivel de dominio está en relación directa con el hecho de haber recibido formación continua o no sobre el particular, en especial en aquellas competencias asociadas a la docencia y la investigación.

De aquí se entiende la reiterada necesidad de formación pedagógica urgente que demanda el profesorado, así como la necesidad de soporte y acompañamiento continuos para la integración adecuada de las TIC en la actividad docente universitaria (Cabero, 2014; Durán, Gutiérrez \& Prendes, 2016; Jakstiene, 2011; Prendes, Gutiérrez \& Martínez, 2018; Sangrà \& González, 2004; Tejada, 2014; Urbina \& Salinas, 2014).

A la par, consideramos que la competencia digital entraña un proceso de aprendizaje complejo, gradual y recurrente, que comprende la capacidad utilizar adecuadamente las herramientas y recursos digitales para buscar, gestionar, analizar y transformar la información en conocimiento de manera crítica, así como la capacidad de trabajar en equipo y compartir dicho conocimiento con ética y responsabilidad social (Área, Gros \& García-Quismondo, 2008; Domínguez et al. 2014; Tannenbaum, y Katz, 2008; UNESCO, 2008 y 2011; Vivancos, 2008). Por ello, consideramos de extrema relevancia que el profesorado reflexione profundamente e investigue sobre ello, en la práctica y para la práctica, y al mismo tiempo, que se tomen las medidas necesarias para repensar y rediseñar la formación de los docentes hacia un aprendizaje continuo, con experiencias de aprendizaje centradas en las problemáticas cotidianas de la enseñanza superior y en integrar significativamente las tecnologías para contribuir a resolverlas. 
De ahí también, en línea con Moser (2014), es imprescindible que la institución universitaria establezca un marco de condiciones pertinentes para que el profesorado use adecuadamente la tecnología. Ello no solo incluye estructuras formales de incentivos, carrera profesional, sino las estructuras tecnológicas y de recursos, pero acompañadas de la formación docente-pedagógica, condiciones básicas para la integración de las TIC y el desarrollo de la competencia digital anteriormente expuestos (Mercader y Pozos, 2015; Prendes, 2010; Robbins y Judge, 2013).

\section{REFERENCIAS}

Adams Becker, S., Cummins, M., Davis, A., Freeman, A., Hall Giesinger, C., and Ananthanarayanan, V. (2017). The NMC Horizon Report: 2017 Higher Education Edition. Austin: The New Media Consortium

Adell, J. (2008). Actividades didácticas para el desarrollo de la competencia digital. Conferencia en el marco de las VII Jornadas de experiencias de innovación educativa de Gipuzkoa: desarrollando competencias. Donostia, 18 de abril.

Ala-Mutka, K. (2011). Mapping Digital Competence: Towards a Conceptual Understanding. JRC 67075 - Joint Research Centre - Institute for Prospective Technological Studies. http://ftp.jrc.es/EURdoc/JRC67075_EN.pdf

Almerich, G., Suárez, J.M., Jornet, J.M. \& Orellana, N. (2011). Las competencias y el uso de las TIC por el profesorado: estructura dimensional. Revista Electrónica de Investigación Educativa, 13(1). Disponible en: http://redie. uabc.mx/vol13no1/contenido-almerichsuarez.html.

Álvarez Rojo, V. Asensio, I.; Clares, J.; del Frago , R.; García Lupión, B.; García Nieto; N. ... Salmerón, P. (2009). Perfiles y competencias docentes requeridos en el contexto actual de la educación universitaria. REOP, 20 (3), $270-283$

Área, M., Gros, B. \& García-Quismondo, M. A. (2008). Alfabetizaciones y tecnologías de la información y comunicación. Madrid: Síntesis

Barroso, J., Cabero, J. \& Vázquez, A. (2012). Formación desde la perspectiva de los entornos personales de aprendizaje. Apertura, Recuperado el 18/03/2012 de: http:/www.udgvirtual.udg.mx/apertura/index.php/apertura3/ article/view/209

Cabero, J. \& Marín, V. (2012). La capacitación en TIC del profesorado universitario en un Entorno Personal de Aprendizaje. El Proyecto DIPRO 2.0. New approaches in Educational Research, 2-7. (http://naerjournal.ua.es/article/view/9).

Cabero, J. (2009). Los nuevos escenarios y las nuevas modalidades de la formación: las aportaciones desde las nuevas y antiguas tecnologías. En: J. Tejada (Coord.) (2009). Estrategias de Innovación en la formación para el trabajo. (pp. 187-208). Madrid: Tornapunta Ediciones.

Cabero, J. (2013). Nuevos escenarios de formación y las nuevas modalidades de formación: el entorno Dipro 2.0. En C. Ruiz et al. (Coords.) Formación para el trabajo en tiempo de crisis. Balance y prospectiva. (pp. 221-231). Madrid: Tornapunta Ediciones.

Cabero, J. (2014). Formación del profesorado universitario en TIC. Aplicación del método Delphi para la selección de los contenidos formativos. Educación XX1, 17 (1), 109-132. https://doi.org/10.5944/educxx1.17.1.10707.

Carrera, F. X. \& Coiduras, J. L. (2012). Identificación de la competencia digital del profesor universitario: un estudio exploratorio en el ámbito de las ciencias sociales. REDU - Revista de Docencia Universitaria. 10 (2), pp. 273 298. https://doi.org/10.4995/redu.2012.6108 
Castañeda, L. \& Adell, J. (Eds.) (2013). Entornos Personales de Aprendizaje: claves para el ecosistema educativo en red. Alcoy: Marfil.

Cebrián, M. (2013). OLË con las tecnologías de federación: Gteavirtual. En C. Ruiz et al. (Coords.) Formación para el trabajo en tiempo de crisis. Balance y prospectiva. (pp. 233-239). Madrid: Tornapunta Ediciones.

Del Moral, M.E. \& Villalustre, L. (2012). Didáctica universitaria en la era 2.0: competencias docentes en campus virtuales. RUSC. Revista de Universidad y Sociedad del Conocimiento, 9 (1), 36-50. https://doi.org/10.7238/rusc.v9i1.1127

Domínguez, J.A. Bárcenas, J. Ruiz-Velasco, S. Tolosa, J.S. (2014). Hacia las competencias digitales en la actividad docente: un diagnóstico para definir los elementos fundamentales. XV Encuentro Internacional Virtual Educa Perú 2014, Lima, 9-13 de junio 2014. http://www.virtualeduca.org/ponencias2014/120/Hacialascompetenciasdigitales.pdf

Durán, M.; Gutiérrez, I. \& Prendes, M.P. (2016). Análisis conceptual de modelos de competencia digital del profesorado universitario. RELATEC Revista Latinoamericana de Tecnología Educativa, 15(1), 97-114. https://doi. org/10.17398/1695288X.15.1.97

Fandos, M. (2013). Los nuevos escenarios y las nuevas modalidades de formación. En C. Ruiz et al. (Coords.) Formación para el trabajo en tiempo de crisis. Balance y prospectiva. (pp. 251-261). Madrid: Tornapunta Ediciones.

Fernández de la Iglesia, J.C. (2012). Competencias TIC de los docentes para la sociedad del conocimiento. Universidad de Santiago de Compostela, tesis doctoral inédita.

Ferrari, A. (2012). Digital Competence in Practice: An Analysis of Frameworks. JRC 25351 - Joint Research Centre Institute for Prospective Technological Studies. http://ftp.jrc.es/EURdoc/JRC25351_EN.pdf

Gallego, M.J.; Gámiz, V. \& Gutiérrez, E. (2010) El futuro docente ante las competencias en el uso de las tecnologías de la información y comunicación para enseñar. EDUTEC. Revista Electrónica de Tecnología Educativa, 34, 1-18. http://edutec.rediris.es/revelec2/revelec34/

González Soto, A.P. (2009). Las tecnologías de la información y la comunicación (TIC) y del aprendizaje y la comunicación (TAC) y revolución formativa. En: J. Tejada (Coord.) (2009). Estrategias de Innovación en la formación para el trabajo. (pp. 89-104). Madrid: Tornapunta Ediciones.

Gutiérrez, I. (2014). Perfil del profesor universitario español en torno a las competencias en TIC. Pixel-Bit. Revista de Medios y Educación, 44, 51-65. https://doi.org/10.12795/pixelbit.2014.i44.04

Hernández Sampieri, R.; Fernández Collado, C. \& Baptista Lucio, P. (2014). Metodología de la Investigación (6ª ed.). México: Mc Graw-Hil.

INTEF-MECD (2013). Marco Común de Competencia Digital Docente. Disponible http://educalab.es/ documents/10180/12809/MarcoComunCompeDigiDoceV2.pdf/e8766a69-d9ba-43f2-afe9-f526fOb34859

ISTE (International Society for Technology in Education) (2008) NETS for Teachers:National Educational Technology Standards for Teachers, Second Edition, http://www.iste.org

Jakstiene, V. (2011). The Coherence of Teacher's ICT Competence and Study Programmes. Social Sciences, 71(1), 62-72. https://doi.org/10.5755/j01.ss.71.1.384

Johnson, L., Adams Becker, S., Estrada, V., \& Freeman, A. (2015). NMC Horizon Report: Edicación Educación Superior 2015. Austin: The New Media Consortium.

Mas, O. \& Tejada J. (2013). Funciones y competencias en la docencia universitaria. Madrid: Síntesis Ed.

Mateo \& Martínez, (2008). Medición y Evaluación Educativa. Madrid: La Muralla.

Mayorga, M.J. \& Núnez, F. (2011). La competencia digital de los docentes: formación y actualización en web 2.0. Etic@ net, IX (11) 213-232.

Medina-Talavera, J.A. (2014). Competencias en las nuevas tecnologías requeridas por los docentes. Saarbrücken (Alemania): Publicia

MEN Ministerio de Educación Nacional (2013). Competencias Tic para el desarrollo profesional docente. Bogotá (Colombia): Imprenta Nacional.

Mengual, S. \& Roig, R. (2012). La enseñanza y las competencias TIC en el contexto universitario. En Y. Sandoval, A. Arenas, E. López, J. Cabero \& J.J. Aguaded (coords.). Las tecnologías de la información en los contextos educativos: 
Nuevos escenarios de aprendizaje. (pp. 17-32). Santiago de Cali: Universidad Santiago de Cali.

Mercader, C. \& Pozos, K.V. (2015). Conditioning factors for pedagogical integration of technologies in higher education teaching: a cross-case analysis of two studies in Mexico and Spain. EDULEARN15 Proceedings Book of the 7th International Conference on Education and New Learning Technologies. Barcelona, Spain: IATED. 6-8 July, 2015.

Moser, F.Z. (2007). Faculty Adoption of Educational Technology. Educause Quarterly, 30(1), 66-69.

Navío, A. (2005). Las competencias profesionales del formador. Barcelona: Octaedro.

Newrly, P. \& Veugelers, M. (2009). How to strengthen digital literacy? Practical example of a European initiative “SPreaD". eLearning Papers, 12.

Pozos, K. V.(2010). La competencia digital del profesorado universitario para la sociedad delconocimiento. Aproximación de un modelo y validación de un cuestionario de detección de necesidades de formación continua. Bellaterra: Dpto. Pedagogia Aplicada. UAB.

Pozos, K.V. (2011). Tecnologías emergentes, competencias digitales relevantes para el profesorado universitario en la sociedad del conocimiento. Jornadas Universitarias de Tecnología Educativa. Sevilla

Pozos, K. V. (2013). Digital competence of Higher Education Teachers for KnowledgeSociety: A Preliminary approach of a Need Assessment Study in Mexican Universities. ICERI2013 -6th International Conference of Education, Research and Innovation. International Association of Technology, Education and Development,

Pozos, K. V. (2015). Evaluación de necesidades de formación continua en Competencia Digital del Profesorado Universitario mexicano para la Sociedad del Conocimiento. Tesis Doctoral, Depto. Pedagogia Aplicada, Universitat Autònoma de Barcelona.

Prendes, M.P. \& Gutiérrez, I. \& Martínez, F. (2018). Competencia digital: una necesidad del profesorado universitario en el siglo XXI. RED. Revista de Educación a Distancia, 56, Artíc. 7. https://doi.org/10.6018/red/56/7.

Prendes, M.P. \& Gutiérrez, I. (2013). Competencias tecnológicas del profesorado en las universidades españolas. Revista de Educación, 361, 196-222. https://doi.org/10-4438/1988-592X-RE-2011-361-140

Prendes, M.P. (Dir.) (2010). Competencias TIC para la docencia en la universidad pública española: Indicadores y propuestas para la definición de buenas prácticas. Informe del proyecto Estudio y Análisis (MEC) (EA2009. 0133). http://www.um.es/competenciastic/informe_final_competencias2010.pdf

Prendes, M.P.; Castañeda, L. \& Gutiérrez, L. (2010) Competencias para el uso de TIC de los futuros maestros. Comunicar, XVIII (35) 175-182)

Ramírez, M. S. (Coord) (2013). Competencias Docentes y Prácticas Educativas Abiertas en Educación a Distancia. México: Lulu ed.

Recomendación 2006/962/CE del Parlamento Europeo y del Consejo, de 18 de diciembre de 2006, sobre las competencias clave para el aprendizaje permanente [Diario Oficial L 394 de 30.12.2006]. Disponible (01/07/13) en: http:// europa.eu/legislation_summaries/education_training_youth/lifelong_learning/c11090_es.htm

Robbins, S.P; \& Judge, T.A (2013). Organizational Behaviour. New Jersey: Pearson Education Inc.

Roig, R. \& Pascual, A.M. (2012). Las competencias digitales de los futuros docentes. Un análisis con estudiantes de magisterio de educación infantil de la Universidad de Alicante. @tic. Revista d'innovació educativa, 9, 53-60.

Sangrà. A. \& González, M. (Coords.) (2004). La transformación de las universidades a través de las TIC. Discursos y prácticas. Barcelona: Editorial UOC

Suárez-Rodríguez, J.M.; Almerich, G.; Gargallo, B. \& Aliaga, F.M. (2013) Las competencias del profesor en TIC: estructura básica. Educación XX1, 16 (1), 39-62. https://doi.org/10.5944/educxx1.16.1.716

Tannenbaum, R. \& Katz, I. (2008). Setting Standards on the Core and Advanced iSkills ${ }^{\mathrm{Tm}}$ Assessments. Research memorandum. Princeton, NJ: ETS.

Tejada, J. \& Giménez, V. (2007). Formación de formadores. Escenario institucional. Madrid: Thomson.

Tejada, J. \& Pozos, K.V. (2018). Nuevos escenarios y competencias digitales docentes: Hacia la profesionalización docente con TIC. Profesorado. Revista de Currículum y Formación de Profesorado. 22(1), 25-51 
Tejada, J. (2007). Estrategias formativas en contextos no formales orientadas al desarrollo socioprofesional, Revista Iberoamericana de Educación, 43/6, 1-12.

Tejada, J. (2009). Competencias docentes. Profesorado. Revista de Currículum y Formación del profesorado. 13(2), 1-15.

Tejada, J. (2011). Evaluación del desarrollo profesional docente basado en competencias, en C. Marcelo (Coord.). Evaluación del desarrollo profesional docente (pp. 23-47). Barcelona: Editorial Davinci.

Tejada, J. (2012). La alternancia de contextos para la adquisición de competencias profesionales en escenarios complementarios de educación superior: marco y estrategia. Educación XX1, 15 (2), 17-40. https://doi. org/10.5944/educxx1.15.2.125

Tejada, J. (2013). La formación de competencias profesionales a través del aprendizaje servicio. Cultura y educación, 25 (3) 285-294. https://doi.org/10.1174/113564013807749669.

Tejada, J. (2014). Formando formadores: nuevos escenarios y competencias digitales docentes. En V. Marín (Coord.). El hoy y el mañana junto a las TIC. (pp. 94 - 154). Sevilla: Secretariado de Medios Audivisuales y NNTTT, Universidad de Sevilla-EDUTEC,

Tejedor, J. \& García-Valcárcel, A. (2006). Competencias de los profesores para el uso de las TIC en la enseñanza. Análisis de sus conocimientos y actitudes. Revista Española de Pedagogía, 223, 21-44.

Tójar, C. (2006). Investigación cualitativa. Comprender y actuar. Madrid: La Muralla.

UNESCO (2008). Estándares de competencia en TIC para docentes. Paris: UNESCO.

UNESCO (2011). UNESCO ICT competency framework for teachers. Paris: UNESCO.

Urbina, S. \& Salinas, J. (2014). Campus virtuales: una perspectiva evolutiva y tendencias. RED. Revista de Educación a Distancia, 42, http://www.um.es/ead/red/42

Vivancos, J. (2008). Tratamiento de la información y competencia digital. Madrid: Alianza Editorial.

RIDU / Revista Digital de Investigación en Docencia Universitaria / ISNN 2223-2516

(c) Los autores. Este artículo es publicado por la Revista Digital de Investigación en Docencia Universitaria del Área de Institutional Research and Effectiveness de la Dirección de Aseguramiento de la Calidad, Universidad Peruana de Ciencias Aplicadas. Este es un artículo de acceso abierto, distribuido bajo los términos de la LicenciaCreativeCommons Atribución-CompartirIgual 4.0 Internacional. ( http://creativecommons.org/licenses/by-sa/4.0/), que permite el uso no comercial, distribución y reproducción en cualquier medio, siempre que la obra original sea debidamente citada. 


\section{APÉNDICE A.}

\section{Matriz de Competencias Digitales del Profesorado para la Sociedad del Conocimiento (Pozos Pérez, 2013)}

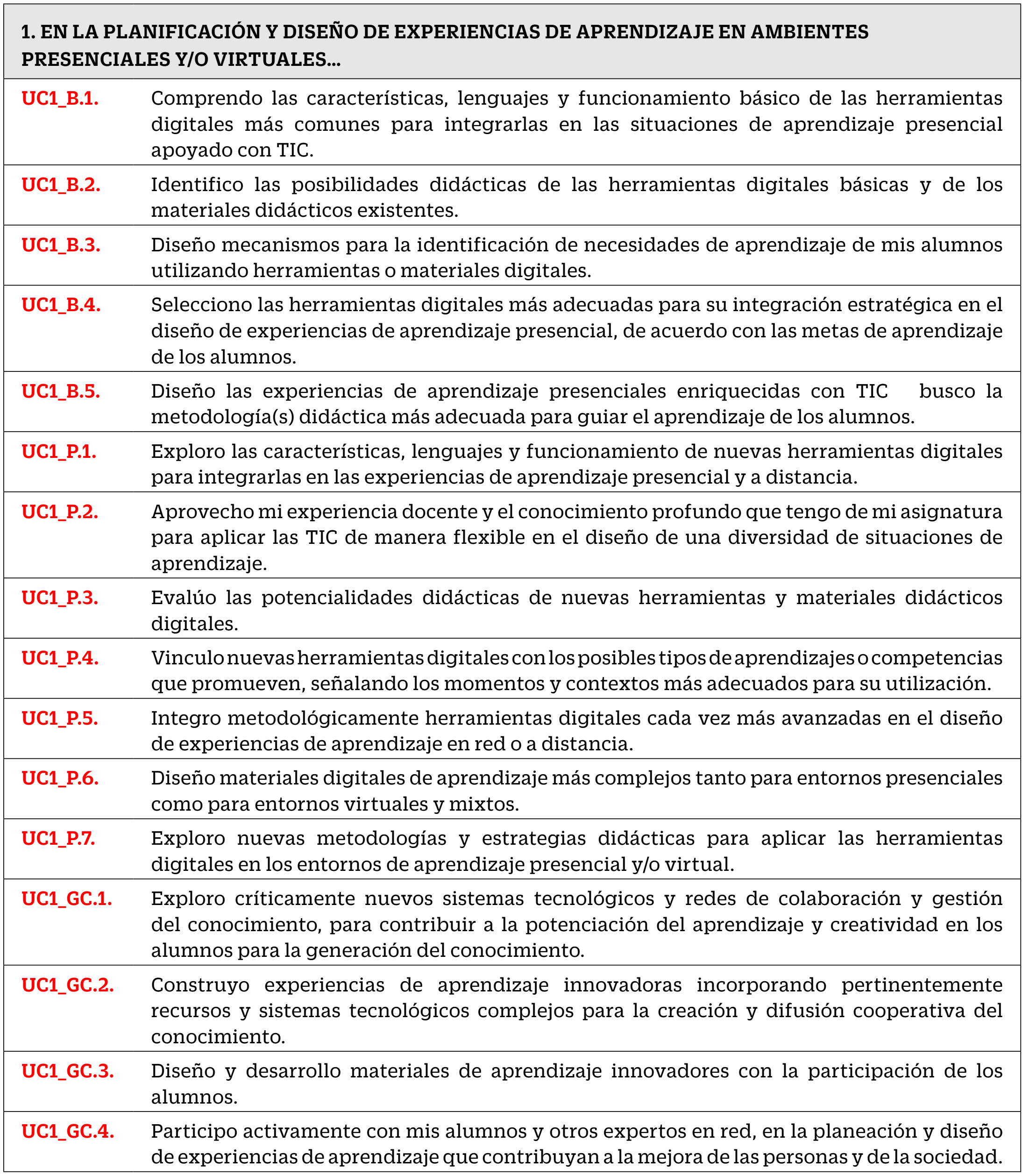




\section{EN EL DESARROLLO Y CONDUCCIÓN DE EXPERIENCIAS DE APRENDIZAJE COLABORATIVAS PRESENCIALES Y/O EN RED...}

UC2_B.1. Gestiono experiencias creativas de aprendizaje en entornos presenciales con apoyo de las herramientas digitales básicas para motivar y guiar el aprendizaje de los alumnos.

UC2_B.2. Utilizo las metodologías de enseñanza - aprendizaje que conozco hasta el momento, para integrar las herramientas digitales básicas en los momentos adecuados del acto didáctico.

UC2_B.3. Fomento la comunicación y el trabajo colaborativo a través de las herramientas digitales básicas de comunicación y colaboración digital.

UC2_B.4. Gestiono los proyectos de investigación y trabajos de los alumnos con el apoyo de las herramientas digitales básicas.

UC2_P.1. Gestiono ambientes y plataformas de aprendizaje en red para desarrollar nuevas experiencias colaborativas de aprendizaje, que permitan a los alumnos resolver situaciones complejas ya sea simuladas o de la realidad.

UC2_P.2. Adaptoestratégica y metodológicamenteherramientas digitales y materiales deaprendizaje más complejos, para su utilización en los momentos adecuados del acto didáctico.

UC2_P.3. Conduzco los proyectos de investigación y las experiencias de los alumnos a través de casos o problemas simulados o reales, con el apoyo de herramientas digitales avanzadas y de trabajo colaborativo en red.

UC2_P.4. Fomento la comunicación, la reflexión crítica y el liderazgo de los alumnos usando herramientas de comunicación y trabajo colaborativo en red.

UC2_GC.1. Desarrollo metodologías innovadoras de enseñanza con herramientas digitales más avanzadas para construir, gestionar y difundir el conocimiento con los alumnos y con otras personas a través de los diversos tipos de redes.

UC2_GC.2. Exploto al máximo las posibilidades de las plataformas de aprendizaje y de gestión del conocimiento en red para potenciar el conocimiento individual y sobre todo colaborativo de los alumnos.

UC2_GC.3. Adapto y creo mis propias plataformas de aprendizaje y de gestión del conocimiento acordes con las necesidades específicas de los alumnos.

UC2_GC.4. Dirijo a los alumnos en una serie de variadas experiencias y escenarios reales y simulados a través de la red, que le faciliten su propia construcción del conocimiento y la creación y conducción de sus propios proyectos de aprendizaje y de investigación. 


\section{EN CUANTO A LA ORIENTACIÓN, GUÍA Y EVALUACIÓN DE LOS PROCESOS DE CONSTRUCCIÓN DEL CONOCIMIENTO DE LOS ALUMNOS EN ENTORNOS PRESENCIALES Y/O VIRTUALES...}

UC3_B.1. Analizo las necesidades de aprendizaje, los conocimientos previos y las motivaciones de los alumnos con el apoyo de herramientas o materiales digitales.

UC3_B.2. Evalúo continuamente los aprendizajes y los procesos de construcción del conocimiento de los alumnos en entornos presenciales de aprendizaje mediados por TIC.

UC3_B.3. Aplico y ajusto los apoyos necesarios para guiar el aprendizaje con apoyo de las TIC en entornos presenciales de aprendizaje.

UC3_B.4. Utilizo las herramientas digitales para la construcción de instrumentos para la evaluación y auto evaluación de los aprendizajes de los alumnos.

UC3_B.5. Utilizo las plataformas virtuales de gestión de contenidos académicos de la institución para administrar el sistema de evaluación del curso y dar seguimiento a los aprendizajes de los alumnos.

UC3_P.1. Propongo actividades y experiencias de aprendizaje motivadoras y complejas con el uso de herramientas digitales para valorar y fomentar el grado de comprensión, creatividad, análisis crítico y pensamiento práctico de los alumnos.

UC3_P.2. Tutorizo los procesos de aprendizaje y de comunicación de los alumnos en entornos virtuales de aprendizaje, cediendo el control al alumno cuando éste es capaz de asumirlo y recuperando el papel de guía cuando así lo precise.

UC3_P.3. Conduzco la participación y las interacciones de los alumnos con los contenidos, los materiales de aprendizaje, los compañeros, otras personas y expertos en red.

UC3_P.4. Proveo a los alumnos el acceso a múltiples perspectivas y contenidos, así como variadas formas de evaluación y retroalimentación mediadas por TIC.

UC3_GC.1. Modelo el pensamiento creativo e innovador impulsando a los alumnos a que participen y se impliquen activamente en la planeación, desarrollo y resolución de situaciones o problemas de la realidad social con el apoyo de las TIC.

UC3_GC.2. Analizo colaborativamente con alumnos y expertos a través de la red, el contexto social actual y sus problemáticas específicas para orientar las reflexiones y los procesos de construcción del conocimiento.

UC3_GC.3. Apoyo el proceso de reflexión de los alumnos cuando se enfrentan a la evaluación externa proveniente de su participación en proyectos de la realidad social. 


\section{EN LA GESTIÓN DE MI CRECIMIENTO Y DESARROLLO PROFESIONAL CON APOYO DE LAS TIC...}

UC4_B.1. Hago uso de las TIC, especialmente en la adquisición de conocimientos complementarios sobre mis asignaturas y sobre pedagogía-didáctica, que contribuyan a mi propio desarrollo profesional.

UC4_B.2. Utilizo las herramientas tecnológicas básicas de gestión académica en los ámbitos profesionales habituales para apoyar mi desempeño profesional.

UC4_B.3. Participo en propuestas de formación básica en/con TIC para el mejoramiento de mi actividad profesional habitual.

UC4_B.4. Valoro la importancia y la necesidad del aprendizaje permanente en diversos ámbitos y en TIC para el mejoramiento de mi desarrollo profesional.

UC4_P.1. Me comunico activamente y comparto el conocimiento con mis alumnos y otros expertos o personas a través de la red.

UC4_P.2. Participo continuamente en diversas propuestas y modalidades de formación en/con TIC ajustadas a mis necesidades y al plan personal de desarrollo profesional.

UC4_P.3. Me comprometo con el aprendizaje a lo largo de la vida para el mejoramiento constante de mi desarrollo profesional y personal, procurando integrar los últimos avances científicos y tecnológicos.

UC4_GC.1. Creo mis propias redes sociales, de aprendizaje, de trabajo y personales a través de la red y lo promuevo en mis alumnos.

UC4_GC.2. Potencio la exploración activa de nuevas formas de crecimiento y desarrollo profesional con otras personas a través de redes sociales y profesionales.

UC4_GC.3. Creo mis propias propuestas y modalidades de formación para el desarrollo y crecimiento profesional de otros profesionales a través de la red y las TIC.

\section{EN LA INVESTIGACIÓN, DESARROLLO E INNOVACIÓN PEDAGÓGICA CON TIC Y PARA EL USO DE LAS TIC EN EDUCACIÓN...}

UC5_B.1. Consulto información relevante a través de las herramientas digitales básicas para el desarrollo de mis actividades profesionales cotidianas de docencia, investigación y gestión.

UC5_B.2. Reflexiono sobre la práctica, acerca de los beneficios de la utilización de las herramientas digitales básicas en las experiencias educativas presenciales.

UC5_B.3. Participo en proyectos de investigación con apoyo de las herramientas digitales básicas.

UC5_P.1. Diseño, desarrollo y evalúo proyectos de investigación e innovación con/para las TIC orientados a la mejora de la práctica profesional.

UC5_P.2. Investigo y aplico reflexivamente, sobre y en la práctica, las posibilidades y beneficios reales de las TIC en los procesos de enseñanza - aprendizaje presencial y virtual. 


\section{EN LA INVESTIGACIÓN, DESARROLLO E INNOVACIÓN PEDAGÓGICA CON TIC Y PARA EL USO DE LAS TIC EN EDUCACIÓN...}

UC5_P.3. Reflexiono constructivamente a través de redes con otros profesores o profesionales expertos, para compartir y debatir sobre el conocimiento didáctico-pedagógico, el conocimiento de la profesión y el contexto social local y global sobre el que actuamos.

UC5_P.4. Difundo el conocimiento a través mi participación en seminarios, congresos, foros de debates y diversas actividades presenciales y virtuales a través de la red.

UC5_GC.1. Propicio la construcción colaborativa del conocimiento comprometiéndome en el aprendizaje con alumnos, colegas y otros en ambientes presenciales y virtuales.

UC5_GC.2. Participo en la construcción del conocimiento pedagógico, en la política curricular y en la toma de decisiones respecto de los procesos educativos con apoyo de las herramientas digitales.

UC5_GC.3. Creo redes virtuales para estimular la innovación y la calidad mediante la reflexión y el intercambio mutuo.

\section{EN CUESTIONES SOBRE DIVERSIDAD, ÉTICA Y USO RESPONSABLE DE LAS TIC EN EL DESEMPEÑO PROFESIONAL DOCENTE...}

UC6_B.1. Comprendo las implicaciones y los riesgos potenciales de las herramientas digitales en la segregación y exclusión social.

UC6_B.2. Actúo con criterios éticos para la integración responsable de las TIC en el currículo y en las actividades de aprendizaje de los alumnos.

UC6_B.3. Garantizo el acceso equitativo al uso de las TIC a los alumnos durante las experiencias de aprendizaje presenciales, especialmente en las actividades colaborativas.

UC6_B.4. Proporciono experiencias y materiales digitales de aprendizaje considerando la diversidad cultural y lingüística de los alumnos.

UC6_P.1. Garantizo el acceso equitativo al uso de las TIC a los alumnos durante las experiencias de aprendizaje virtuales y en red.

UC6_P.2. Personalizo y adapto el diseño de las actividades de aprendizaje virtuales para atender a la diversidad de los alumnos.

UC6_P.3. Fomento el respeto a la diversidad cultural y de diversas formas de pensamiento durante las experiencias de aprendizaje y de comunicación colaborativas en los entornos virtuales de aprendizaje y el uso de redes sociales.

UC6_P.4. Promuevo la libertad de expresión en los procesos comunicativos de los entornos de aprendizaje virtuales.

UC6_P.5. Fomento el uso ético, legal y responsable de las TIC en los alumnos a través del análisis crítico de las implicaciones y consecuencias de su uso inadecuado para la sociedad. 


\section{EN CUESTIONES SOBRE DIVERSIDAD, ÉTICA Y USO RESPONSABLE DE LAS TIC EN EL DESEMPEÑO PROFESIONAL DOCENTE... \\ UC6_GC.1. Promuevo el debate y el análisis crítico sobre el uso ético y aplicación responsable de las TIC y del nuevo conocimiento generado. \\ UC6_GC.2. Reconozco y respeto los derechos intelectuales y de autoría sobre los nuevos conocimientos generados a través de las TIC.}

\section{RESPECTO AL MEDIO AMBIENTE, LA SALUD Y LA SEGURIDAD LABORAL CON EL USO DE LAS TIC EN LA PROFESIÓN DOCENTE...}

UC7_B.1. Manejo adecuadamente el estrés y las emociones en la adquisición de nuevos conocimientos y desarrollo de competencias alrededor de las TIC en mi práctica diaria.

UC7_B.2. Utilizo adecuadamente las TIC conjuntamente con los correspondientes manuales de instalación y uso.

UC7_P.1. Reflexiono sobre las TIC utilizadas en la profesión docente y analizo su impacto global en el medio ambiente.

UC7_P.2. Utilizo adecuadamente las herramientas digitales, en el momento y condiciones adecuados de seguridad tanto para mí como para mis alumnos.

UC7_P.3. Conozco y aplico las normas básicas de seguridad alrededor de las TIC para prevenir riesgos y afecciones de salud en mi práctica docente en general.

UC7_GC.1. Desarrolloestrategias innovadoras para la utilización delas TIC queimpacten positivamente en el medio ambiente.

UC7_GC.2. Oriento la generación del conocimiento para la utilización racional y segura de las TIC en la preservación y mejoramiento del medio ambiente y de las condiciones de salud en la profesión docente. 